\title{
Asymptotics for the Spectrum of the Dirichlet Laplacian on Horn-shaped Regions
}

\author{
M. VAN DEN BERG \& M. LIANANTONAKIS
}

ABSTRACT. Let $\Omega$ be an open bounded set in $\mathbb{R}^{m-1}$ with a piecewise smooth boundary, and star-shaped with respect to $(0, \ldots, 0) \in \mathbb{R}^{m-1}$. We extend a result of G.V. Rozenbljum for the spectrum of the Dirichlet Laplace operator for $\{(x, y) \in$ $\left.\mathbb{R}^{m}: y \in(1+x)^{-\alpha} \Omega, x>0\right\}$ in $\mathbb{R}^{m}$, where $\alpha>0$. For $2 m-1>\alpha>\left(1-m+[(m-1)(9 m-17)]^{1 / 2}\right) / 2$ we obtain two-term asymptotics and a remainder estimate for the Dirichlet counting function. For $\alpha \geq 2 m-1$ or $m>2$ and $(1-m+$ $\left.[(m-1)(9 m-17)]^{1 / 2}\right) / 2 \geq \alpha>0$ we recover Rozenbljum's result for the leading term of the Dirichlet counting function, together with an improved remainder estimate.

\section{INTRODUCTION}

Let $D$ be an open set in euclidean space $\mathbb{R}^{m}$ with boundary $\partial D$, and let $-\Delta_{D}$ be the Dirichlet laplacian for $D$. A theorem of $H$. Weyl [24] asserts that, if $D$ is bounded in $\mathbb{R}^{m}(m=2,3, \ldots)$ and if $\partial D$ is piecewise smooth, then the spectrum of $-\Delta_{D}$ is discrete $\lambda_{1} \leq \lambda_{2} \leq \cdots$. Furthermore, if $N_{D}$ is the counting function defined by

$$
N_{D}(\lambda)=\#\left\{j \in \mathbb{Z}^{+}: \lambda_{j}<\lambda\right\},
$$

then

$$
\lim _{\lambda \rightarrow \infty} \frac{N_{D}(\lambda)}{W_{D}(\lambda)}=1,
$$

where $W_{D}$ is the Weyl function defined by

$$
W_{D}(\lambda)=(4 \pi)^{-m / 2}\left(\Gamma\left(\frac{m}{2}+1\right)\right)^{-1}|D|_{m} \lambda^{m / 2},
$$


and $|D|_{m}$ is the $m$-dimensional Lebesgue measure of $D$.

Weyl's theorem (1.2) has been improved in various directions. First of all, it was shown in [17], [18] that (1.2) holds under the minimal hypothesis $D$ open and $|D|_{m}<\infty$ (see also [20, Theorem 10.6]). Secondly, if $D$ is bounded and $\partial D$ is piecewise smooth, then (1.2) can be improved to

$$
N_{D}(\lambda)=W_{D}(\lambda)+O\left(\lambda^{(m-1) / 2}\right), \quad \lambda \rightarrow \infty,
$$

([14], [19]). If in addition the measure of periodic geodesics in $D$ is equal to zero, then (1.4) can be improved to

$$
\begin{array}{r}
N_{D}(\lambda)=W_{D}(\lambda)-4^{-1}(4 \pi)^{-(m-1) / 2}\left(\Gamma\left(1+\frac{m}{2}\right)\right)^{-1} A(\partial D) \lambda^{(m-1) / 2} \\
+o\left(\lambda^{(m-1) / 2}\right), \quad \lambda \rightarrow \infty,
\end{array}
$$

where $A(\partial D)$ is the area of $\partial D([8],[23])$.

In this paper we consider unbounded open sets in $\mathbb{R}^{m}$ with possibly infinite volume. A necessary and sufficient condition in the infinite volume case for $-\Delta_{D}$ to have discrete spectrum has been obtained in [11]. Though various bounds for $N_{D}$ have been obtained in this case [16], [17], [18], [5], we do not know of any formula which could possibly replace (1.2). In fact, some of the examples in [10] illustrate the very complicated oscillatory asymptotic behaviour that may occur, even for regions in $\mathbb{R}^{2}$. Here we concentrate on a wide class of unbounded hornshaped regions in $\mathbb{R}^{m}$, where detailed asymptotic analysis of $N_{D}(\lambda)$ for $\lambda \rightarrow \infty$ remains possible.

Let $m=2,3, \ldots$ and let $\Omega$ be an open bounded set in $\mathbb{R}^{m-1}$, star-shaped with respect to $(0, \ldots, 0)$. Let $f:[0, \infty) \rightarrow[0,1]$ be monotonically decreasing to zero with $f(0)=1$. Let

$$
D(\Omega, f)=\left\{(x, y) \in \mathbb{R}^{m}: \frac{y}{f(x)} \in \Omega, x>0\right\} .
$$

$D(\Omega, f)$ is an example of a horn-shaped region in $\mathbb{R}^{m}$. It is well-known that the spectrum of $-\Delta_{D(\Omega, f)}$ is discrete. Estimates for the corresponding counting function have been obtained in [4, Theorem 8]. However, in order to analyse the asymptotic behaviour of $N_{D(\Omega, f)}$ and to exclude possible oscillatory behaviour as observed in [10], it is necessary to restrict the class of functions for $f$ even further. In fact, the case where $f=f_{\alpha}, \alpha>0$, and

$$
f_{\alpha}(x)=(1+x)^{-1 / \alpha}, \quad x>0,
$$

subdivides already into many subcases. G.V. Rozenbljum ([17, p. 246], [18]) proved the following. Let $|\Omega|_{m-1}<\infty$ and $\alpha>m-1$. Then for $\lambda \rightarrow \infty$,

$$
N_{D\left(\Omega, f_{\alpha}\right)}(\lambda)=\zeta(\alpha, \Omega) \lambda^{(1+\alpha) / 2}+o\left(\lambda^{(1+\alpha) / 2}\right),
$$


where the spectral zeta function $\zeta(\alpha, \Omega)$ is given by

$$
\zeta(\alpha, \Omega)=(4 \pi)^{-1 / 2} \frac{\Gamma((\alpha+2) / 2)}{\Gamma((\alpha+3) / 2)} \sum_{j=1}^{\infty} \mu_{j}^{-\alpha / 2},
$$

and $\mu_{1}<\mu_{2} \leq \cdots$ is the spectrum of the $(m-1)$-dimensional Dirichlet laplacian $-\Delta_{\Omega}$ for $\Omega$.

The special case where $\Omega=\Omega_{1}$, the unit disc in $\mathbb{R}^{m-1}$, has been investigated by many authors ([3], [4], [9], [13], [21], [22] and the references therein). In particular the case where $m=2$ and $\Omega=(-1,1)$ has received a great deal of attention. In this case $\mu_{j}=(\pi j / 2)^{2}, j \in \mathbb{Z}^{+}$, and the spectral zeta function in (1.9) reduces to a constant multiple of the Riemann zeta function. One easily recovers [21, Theorem 1.3], [3, Examples 1 and 2], and the results of [9, Section 3].

The higher dimensional situation where $\Omega=\Omega_{1}, m=2,3, \ldots$, and $f=f_{\alpha}$ has been investigated in [13] under the hypothesis that the measure of periodic geodesics on $D\left(\Omega_{1}, f_{\alpha}\right)$ is equal to zero (billiard conditions). The author obtained the structure of the asymptotic behaviour for the corresponding counting function, including some results for the finite volume case. However, some of the coefficients in the asymptotic formulae remained undetermined. Moreover, by fixing the geometry of the cross-section $\Omega=\Omega_{1}$, it is not possible to give a geometric interpretation of the coefficients. See also the remark on [21, line 4, p. 86].

Estimates for the counting function for elliptic operators of even order with variable coefficients stabilizing at infinity in a regular cusp were obtained by Ivrii in [8, Chapter 12.1]. Some of these results were obtained under the hypothesis that the influence of the periodic bi-characteristics is small (billiard conditions).

In this paper we avoid making the hard-checkable billiard conditions by using the variational method. We obtain upper and lower bounds for the spectral counting function for the Dirichlet laplacian on $D(\Omega, f)$ given by (1.6), where $f$ is an arbitrary to zero decreasing function, and where $\Omega$ is an open, bounded and star-shaped set in $\mathbb{R}^{m-1}$ satisfying mild regularity conditions (Theorem 3.1 and Theorem 5.1). In the special case where $f=f_{\alpha}$, given by (1.7), we refine Rozenbljum's result (1.8), (1.9) by obtaining an explicit expression for the second term in (1.8) (Theorem 1.1). It turns out that if $2 m-1>\alpha>m-1$, then this second term is a multiple of $|\Omega|_{m-1} \lambda^{m / 2}$. This provides the geometric interpretation, which was lacking in the earlier results. In addition we obtain for $2 m-1>\alpha>m-1$ non-trivial remainder estimates. For the finite volume case $m-1>\alpha$ we show that the leading term is given by the corresponding Weyl function, whereas the second term is a multiple of $\lambda^{(1+\alpha) / 2}$. The coefficient involves a regularized zeta function. 
Theorem 1.1. Suppose that $\Omega$ in $\mathbb{R}^{m-1}$ is an open, bounded set and star-shaped with respect to 0 . For $m=3,4, \ldots$ we also assume that $\partial \Omega$ is piecewise smooth. Let

$$
D\left(\Omega, f_{\alpha}\right)=\left\{(x, y) \in \mathbb{R}^{m-1}:(1+x)^{1 / \alpha} y \in \Omega, x>0\right\} .
$$

Then for $\lambda \rightarrow \infty$ and

(i) $\alpha \geq 2 m-1$

$$
N_{D\left(\Omega, f_{\alpha}\right)}(\lambda)=\zeta(\alpha, \Omega) \lambda^{(1+\alpha) / 2}+O\left(\lambda^{(1+\alpha) / 4} \log \log \lambda\right),
$$

(ii) $2 m-1>\alpha>2 m-2$

$$
-\frac{\alpha}{\alpha+1-m}(4 \pi)^{-m / 2}\left(\Gamma\left(\frac{m}{2}+1\right)\right)^{-1}|\Omega|_{m-1} \lambda^{m / 2}+O\left(\lambda^{(1+\alpha) / 4} \log \log \lambda\right),
$$

(iii) $2 m-2 \geq \alpha>m-1$

(1.13) $N_{D\left(\Omega, f_{\alpha}\right)}(\lambda)=\zeta(\alpha, \Omega) \lambda^{(1+\alpha) / 2}$

$$
-\frac{\alpha}{\alpha+1-m}(4 \pi)^{-m / 2}\left(\Gamma\left(\frac{m}{2}+1\right)\right)^{-1}|\Omega|_{m-1} \lambda^{m / 2}+O\left(\lambda^{(2 m-1) / 4} \log \lambda\right),
$$

(iv) $\alpha=m-1$

(1.14) $\quad N_{D\left(\Omega, f_{m-1}\right)}(\lambda)$

$$
\begin{aligned}
& =\frac{m-1}{2}\left(\Gamma\left(\frac{m}{2}+1\right)\right)^{-1} \lambda^{m / 2} \\
& \cdot\left((4 \pi)^{-m / 2}|\Omega|_{m-1}\left[\log \lambda-(-1)^{m} \log 4-2 \sum_{k=1}^{m} \frac{(-1)^{k+m}}{k}-\log \mu_{1}\right]\right. \\
& \left.\quad+(4 \pi)^{-1 / 2} \Gamma\left(\frac{m+1}{2}\right) \int_{\left[\mu_{1}, \infty\right)} \mu^{-(m+1) / 2}\left(N_{\Omega}(\mu)-W_{\Omega}(\mu)\right) d \mu\right) \\
& +O\left(\lambda^{(3 m-2) / 6} \log \lambda\right),
\end{aligned}
$$

(v) $m-1>\alpha>\left(1-m+[(m-1)(9 m-17)]^{1 / 2}\right) / 2$

(1.15) $\quad N_{D\left(\Omega, f_{\alpha}\right)}(\lambda)=W_{D\left(\Omega, f_{\alpha}\right)}(\lambda)+\eta(\alpha, \Omega) \lambda^{(1+\alpha) / 2}$

$$
+O\left(\lambda^{\left(m \alpha+2 m^{2}-4 m+2\right) /(2 \alpha+4 m-4)} \log \lambda\right),
$$


(vi)

$$
\left(1-m+[(m-1)(9 m-17)]^{1 / 2}\right) / 2 \geq \alpha>0
$$

$$
N_{D\left(\Omega, f_{\alpha}\right)}(\lambda)=W_{D\left(\Omega, f_{\alpha}\right)}(\lambda)+O\left(\lambda^{\left(m \alpha+2 m^{2}-4 m+2\right) /(2 \alpha+4 m-4)} \log \lambda\right),
$$

where the spectral zeta function $\zeta(\alpha, \Omega)$ in (1.11)-(1.13) is given by (1.9), the regularized spectral zeta function $\eta(\alpha, \Omega)$ in (1.15) is given by

$$
\eta(\alpha, \Omega)=(4 \pi)^{-1 / 2} \frac{\alpha \Gamma((\alpha+2) / 2)}{2 \Gamma((\alpha+3) / 2)} \int_{[0, \infty)} \mu^{-(2+\alpha) / 2}\left\{N_{\Omega}(\mu)-W_{\Omega}(\mu)\right\} d \mu,
$$

and the Weyl function $W_{D\left(\Omega, f_{\alpha}\right)}$ in (1.15) and (1.16) is given by (1.3), with

$$
\left|D\left(\Omega, f_{\alpha}\right)\right|_{m}=\frac{\alpha}{m-1-\alpha}|\Omega|_{m-1} .
$$

Remark 1.2.

(i) We note the very complicated coefficient of $\lambda^{m / 2}$ in borderline case $\alpha=$ $m-1$ in (1.14). The role of the principal eigenvalue $\mu_{1}$ of $-\Delta_{\Omega}$ in that coefficient is not special: the coefficient remains unchanged if we replace $\mu_{1}$ by any $\varepsilon \in\left(0, \mu_{1}\right]$.

(ii) We note that for $m=2$, case (vi) becomes vacuous and the remainder in $(1.15)$ is $O\left(\lambda^{(1+\alpha) / 2}\right)$. This implies two-term asymptotics for all finite volume cases $1>\alpha$. In fact, case (vi) only occurs because the remainder estimate in (1.15) dominates the second term $\lambda^{(1+\alpha) / 2}$ for $m>2$.

(iii) Assuming billiard conditions, it is possible, by using results of [8, Chapter 12.1], to improve the remainder estimates for $m-1>\alpha$. For $m-1>\alpha>$ $m-2$ one recovers two-term asymptotics with the second term as in (1.15), whereas for $m-2>\alpha>0$ one recovers (1.5).

Theorem 1.1 implies the following improvement of [4, Corollary 3].

Corollary 1.2. Let $D_{\alpha} \subset \mathbb{R}^{2}$ be given by

$$
D_{\alpha}=\left\{\left(x_{1}, x_{2}\right) \in \mathbb{R}^{2}:\left|x_{1}\right| \cdot\left|x_{2}\right|^{\alpha}<1\right\} .
$$

Then for $\lambda \rightarrow \infty$,

$$
\begin{aligned}
& N_{D_{\alpha}}(\lambda)=\beta(\alpha) \lambda^{(1+\alpha) / 2}+\beta\left(\frac{1}{\alpha}\right) \lambda^{(1+\alpha) /(2 \alpha)}+O\left(\lambda^{(1+\alpha) / 4} \log \lambda\right), \\
& N_{D_{\alpha}}(\lambda)=\beta(\alpha) \lambda^{(1+\alpha) / 2}+O\left(\lambda^{(1+\alpha) / 4} \log \lambda\right), \quad \alpha \geq 2, \\
& N_{D_{1}}(\lambda)=\pi^{-1} \lambda \log \lambda+2 \pi^{-1}(\gamma-\log \pi) \lambda+O\left(\lambda^{2 / 3} \log \lambda\right),
\end{aligned}
$$


where

$$
\beta(\alpha)=2^{\alpha} \pi^{-\alpha-1 / 2} \zeta(\alpha) \frac{\Gamma((\alpha+2) / 2)}{\Gamma((\alpha+3) / 2)},
$$

and $\zeta(\alpha)$ is the Riemann zeta function defined on $\mathbb{C} \backslash\{1\}$. The constant $\gamma$ in (1.22) is Euler's constant.

We note that (1.20) gives two-term asymptotics for $1<\alpha<2$, whereas [4, Corollary 3] gave two-term asymptotics only for $1<\alpha<2^{1 / 2}$. It is possible to extend Corollary 1.2 to the situation in $\mathbb{R}^{m}$ where $\alpha=\left(\alpha_{1}, \alpha_{2}, \ldots, \alpha_{m}\right), 0<$ $\alpha_{1} \leq \alpha_{2} \leq \cdots \leq \alpha_{m}$, and

$$
D_{\alpha}=\left\{\left(x_{1}, \ldots, x_{m}\right) \in \mathbb{R}^{m}:\left|x_{1}\right|^{\alpha_{1}} \cdots\left|x_{m}\right|^{\alpha_{m}}<1\right\} \text {. }
$$

We will not present the details, except for the following special case.

Theorem 1.3. Let $m=3,4, \ldots, \alpha=(1,1, \ldots, 1)$ and let $D_{\alpha}$ be given by (1.24). Then for $\lambda \rightarrow \infty$ and $m=3,4, \ldots$,

$$
N_{D_{\alpha}}(\lambda)=a_{m} \lambda^{m / 2}(\log \lambda)^{m-1}+b_{m} \lambda^{m / 2}(\log \lambda)^{m-2}+O\left(\lambda^{m / 2}(\log \lambda)^{m-3}\right),
$$

where

$$
a_{m}=\frac{2^{(m-1)(m-2) / 2}}{\pi^{m / 2} \Gamma(1+m / 2)}
$$

and where the constants $b_{m}$ are recursively defined by

$$
\begin{gathered}
b_{m}=2^{\left(m^{2}-3 m+4\right) / 2} \frac{m-1}{\Gamma(m / 2+1)} \pi^{-m / 2} \\
\cdot\left\{(-1)^{m+1} \log 2+\sum_{k=1}^{m} \frac{(-1)^{k+m+1}}{k}\right\} \\
+2^{m-2} \frac{m-1}{m-2} \frac{\Gamma((m+1) / 2)}{\Gamma(m / 2+1)} \pi^{-1 / 2} b_{m-1}, \\
b_{2}=\frac{2}{\pi}(\gamma-\log \pi) .
\end{gathered}
$$

The proof of Theorem 1.1 is organized as follows. For $\alpha<m-1$ (the finite volume case) we let $L>0$ be large and partition $D\left(\Omega, f_{\alpha}\right)$ into a "thick end" $D_{L}^{1}$, and into a "horn-shaped end" $D_{L}^{2}$ :

$$
D_{L}^{1}=D\left(\Omega, f_{\alpha}\right) \cap\{x<L\}, \quad D_{L}^{2}=D\left(\Omega, f_{\alpha}\right) \cap\{x>L\} .
$$


At the "thick end" it is advantageous to use a Whitney partition of $D_{L}^{1}$ into cubes, while at the horn-shaped end it is advantageous to use a partition of $D_{L}^{2}$ into cuboids of constant width. Since $\partial \Omega$ is piecewise smooth, the area of $D_{L}^{1}$ is at most $O(L)$, for large $L$. Hence by the Courant-Hilbert estimates ([6, VI.4]),

$$
N_{D_{L}^{1}}(\lambda)=W_{D_{L}^{1}}(\lambda)+O\left(L \lambda^{(m-1) / 2} \log \lambda\right) .
$$

To estimate $N_{D_{L}^{2}}$ we use [4, Theorem 8] with $f_{\alpha}^{L}(x)=f_{\alpha}(x+L)$. We then choose $L$ to be $\lambda$-dependent, in order to minimize the sum of the various remainders. Since $L$ is $\lambda$-dependent one has to replace (1.30) by a uniform estimate. This will be done in Section 2 where we also obtain uniform bounds for open sets $D$ in $\mathbb{R}^{m}$ which satisfy

$$
\omega(\varepsilon) \leq C \varepsilon^{m-d}, \quad 0<\varepsilon<\varepsilon_{0},
$$

for some $C<\infty, d \in[m-1, m), \varepsilon_{0}>0$, and where $\omega$ is defined by

$$
\omega(\varepsilon)=\left|\left\{x \in \mathbb{R}^{m}: \operatorname{dist}(x, \partial D)<\varepsilon\right\}\right|_{m} .
$$

In Section 3 we combine the estimates of Section 2 and the estimates for hornshaped regions to obtain an estimate for $N_{D(\Omega, f)}(\lambda)$ (Theorem 3.1). Theorem 1.1 (v) and (vi) follow then by substituting $f=f_{\alpha}$ and by minimizing the remainder with respect to $L$. The proof of Theorem 1.1 (iv) $(\alpha=m-1)$ also relies upon Theorem 3.1 and will be given in Section 4.

For $\alpha>m-1$ it turns out that the partition of $D_{L}^{1}$ into Whitney cubes does not pay off. However, it does pay off to partition $D\left(\Omega, f_{\alpha}\right)$ into a large $\lambda$ dependent number of horn-shaped pieces. The remainder term is minimized by choosing the width in each piece appropriately (Section 5).

The proof of Corollary 1.2 follows the details of the proof of [4, Corollary 3] with estimates (40), (43) being replaced by (1.14), (1.15) for $m=2$ respectively. We omit the details.

The proof of Theorem 1.3 exploits the recursive structure of $D_{\alpha}$ : the crosssection at $x_{1}$ is a region of the form $D_{\alpha}$ in $\mathbb{R}^{m-1}$ where $\alpha=(1, \ldots, 1)$, and $\lambda_{k}(x)=x^{2 /(m-1)} \lambda_{k}(1)$, where $\lambda_{k}(1)$ are the eigenvalues corresponding to the region $D_{\alpha}$ in $\mathbb{R}^{m-1}$. The two-dimensional result (1.22) and minor modifications of the estimates of [4, Theorem 8] complete the proof. We omit the details.

\section{Courant And Hilbert Remainder estimates}

In this section we prove some uniform bounds on the remainder term for the spectral counting function for open sets with finite volume in $\mathbb{R}^{m}$. We carefully keep track of all the geometric constants in these bounds, refining some of the results in [6, Chapter VI.4] and [12]. 
Let $D$ be an open set in $\mathbb{R}^{m}$ with finite volume $|D|_{m}$. For $\varepsilon>0$ we define

$$
\mu(\varepsilon)=|\{x \in D: \operatorname{dist}(x, \partial D)<\varepsilon\}|_{m} .
$$

Theorem 2.1. Suppose there exist constants $d \in[m-1, m)$ and $C<\infty$ such that

$$
\mu(\varepsilon) \leq C \varepsilon^{m-d}, \quad \varepsilon>0 .
$$

Then for $d=m-1$ and

$$
\lambda \geq 4|D|_{m}^{-2 / m}
$$

we have

$$
N_{D}(\lambda) \geq W_{D}(\lambda)-3 C \lambda^{(m-1) / 2} \log \left\{\lambda(2|D| m)^{2 / m}\right\},
$$

and for $d \in(m-1, m)$ and $\lambda>0$ we have

$$
N_{D}(\lambda) \geq W_{D}(\lambda)-5 C \lambda^{d / 2}\{(m-d)(d-m+1)\}^{-1},
$$

where the Weyl counting function $W_{D}$ is defined by (1.3).

Proof. Let $a>0$ be given by

$$
a^{m}=2|D|_{m},
$$

and form the Whitney decomposition of $D$ into hypercubes of size $\left\{a 2^{-p}: p=\right.$ $0,1,2, \ldots\}$. Let $n_{p}$ denote the number of cubes of size $a 2^{-p}$ contained in $D$. Given $p \in \mathbb{N}$, the hypercubes of size $\left\{a 2^{-\ell}: \ell=0,1, \ldots, p-1\right\}$ cover the set $\left\{x \in D: \operatorname{dist}(x, \partial D)>a 2^{-(p-1)} \sqrt{m}\right\}$. Hence the hypercubes of size $a 2^{-p}$ are contained in the set $\left\{x \in D: \operatorname{dist}(x, \partial D) \leq a 2^{1-p} \sqrt{m}\right\}$. Hence

$$
n_{p} \leq a^{-m} 2^{m p} \mu\left(a 2^{1-p} \sqrt{m}\right), \quad p=1,2, \ldots
$$

We note that $n_{0}=0$ by (2.6).

Let $N_{a}$ denote the spectral counting function for the Dirichlet laplacian on a hypercube of size $a$ in $\mathbb{R}^{m}$. Then

$$
N_{a}(\lambda)=\#\left\{\left(k_{1}, \ldots, k_{m}\right) \in \mathbb{N}^{m}: \pi^{2}\left\{k_{1}^{2}+\cdots+k_{m}^{2}\right\}<\lambda a^{2}\right\} .
$$

Suppose

$$
\lambda a^{2}>m \pi^{2} .
$$


Counting the lattice points in (2.8) gives, by Gauss' estimate,

$$
\begin{aligned}
N_{a}(\lambda) & \geq 2^{-m}\left|\left\{x \in \mathbb{R}^{m}:|x|<\left(\frac{\lambda a^{2}}{\pi^{2}}\right)^{1 / 2}-m^{1 / 2}\right\}\right|_{m} \\
& =\pi^{m / 2} 2^{-m}\left(\Gamma\left(1+\frac{m}{2}\right)\right)^{-1}\left(\frac{a \lambda^{1 / 2}}{\pi}-m^{1 / 2}\right)^{m} \\
& \geq(4 \pi)^{-m / 2} a^{m} \lambda^{m / 2}\left(\Gamma\left(1+\frac{m}{2}\right)\right)^{-1}\left(1-\frac{\pi m^{3 / 2}}{a \lambda^{1 / 2}}\right) .
\end{aligned}
$$

Note that the right hand side of (2.10) is negative for $0<\lambda a^{2}<m \pi^{2}$, and so (2.10) holds for all $\lambda>0, a>0, m=1,2, \ldots$

By Dirichlet bracketing (see e.g. [15, XIII.15]),

$$
\begin{aligned}
N_{D}(\lambda) & \geq \sum_{p=1}^{\infty} n_{p} N_{a 2^{-p}}(\lambda) \\
& \geq \sum_{\left\{p \in \mathbb{N}: 2^{p}<a \lambda^{1 / 2}\right\}} n_{p} N_{a 2^{-p}}(\lambda) \\
& \geq \sum_{\left\{p \in \mathbb{N}: 2^{p}<a \lambda^{1 / 2}\right\}} n_{p} \frac{a^{m} \lambda^{m / 2} 2^{-m p}}{(4 \pi)^{m / 2} \Gamma(1+m / 2)}\left(1-\frac{\pi m^{3 / 2} 2^{p}}{a \lambda^{1 / 2}}\right) \\
& \geq W_{D}(\lambda)-A_{1}-A_{2},
\end{aligned}
$$

where

$$
\text { (2.13) } \quad A_{2}=\pi m^{3 / 2} \sum_{\left\{p \in \mathbb{N}: 2^{p}<a \lambda^{1 / 2}\right\}} n_{p} \frac{a^{m-1} \lambda^{(m-1) / 2} 2^{-(m-1) p}}{(4 \pi)^{m / 2} \Gamma(1+m / 2)} \text {. }
$$

By (2.7), we obtain for $A_{1}$

$$
A_{1} \leq \sum_{\left\{p \in \mathbb{N}: 2^{p} \geq a \lambda^{1 / 2}\right\}} \frac{\lambda^{m / 2} \mu\left(a 2^{1-p} \sqrt{m}\right)}{(4 \pi)^{m / 2} \Gamma(1+m / 2)},
$$

and by (2.2),

$$
\begin{aligned}
A_{1} & \leq \sum_{\left\{p \in \mathbb{N}: 2^{p} \geq a \lambda^{1 / 2}\right\}} \frac{C \lambda^{m / 2} a^{m-d}(4 m)^{(m-d) / 2}}{(4 \pi)^{m / 2} \Gamma(1+m / 2)} 2^{-p(m-d)} \\
& \leq \frac{4 C \lambda^{d / 2} m^{1 / 2}}{(4 \pi)^{m / 2} \Gamma(1+m / 2)(\log 2)(m-d)} .
\end{aligned}
$$


To estimate $A_{2}$ suppose first that $d=m-1$. By (2.2) and (2.7),

$$
A_{2} \leq \sum_{\left\{p \in \mathbb{N}: 2^{p}<a \lambda^{1 / 2}\right\}} \frac{2 C \pi m^{2} \lambda^{(m-1) / 2}}{(4 \pi)^{m / 2} \Gamma(1+m / 2)} .
$$

By (2.3) and (2.6),

$$
\#\left\{p \in \mathbb{N}: 2^{p}<a \lambda^{1 / 2}\right\} \leq \frac{\log \left(a^{2} \lambda\right)}{\log 4} .
$$

Hence

$$
A_{2} \leq \frac{2 C \pi m^{2}}{(4 \pi)^{m / 2} \Gamma(1+m / 2) \log 4} \lambda^{(m-1) / 2} \log \left\{\left(2|D|_{m}\right)^{2 / m} \lambda\right\} .
$$

Inequality (2.4) now follows from (2.11), (2.15), and (2.18). Suppose $m-1<$ $d<m$. By (2.7) and (2.2),

$$
\begin{aligned}
A_{2} & \leq \frac{C \pi m^{3 / 2} a^{m-d-1}(4 m)^{(m-d) / 2} \lambda^{(m-1) / 2}}{(4 \pi)^{m / 2} \Gamma(1+m / 2)} \sum_{\left\{p \in \mathbb{N}: 2^{p}<a \lambda^{1 / 2}\right\}} 2^{(1-m+d) p} \\
& \leq \frac{2 C \pi m^{2}}{(4 \pi)^{m / 2} \Gamma(1+m / 2)} \lambda^{d / 2}\left(2^{1+d-m}-1\right)^{-1} \\
& \leq \frac{2 C \pi m^{2}}{(4 \pi)^{m / 2} \Gamma(1+m / 2)(\log 2)(1+d-m)} \lambda^{d / 2} .
\end{aligned}
$$

Inequality (2.5) follows from (2.11), (2.15), and (2.19), since for $m=1,2, \ldots$,

$$
2 \pi m^{2}+4 m^{1 / 2} \leq 5(4 \pi)^{m / 2} \Gamma\left(1+\frac{m}{2}\right) \log 2 .
$$

Theorem 2.2. Suppose that there exist constants $d \in[m-1, m)$ and $C<\infty$ such that

$$
\omega(\varepsilon) \leq C \varepsilon^{m-d}, \quad 0<\varepsilon \leq|D|_{m}^{1 / m} .
$$

Then for $d=m-1$ and

$$
\lambda \geq 4|D|_{m}^{-2 / m},
$$

we have

$$
N_{D}(\lambda) \leq W_{D}(\lambda)+10^{m} C \lambda^{(m-1) / 2} \log \left\{\lambda\left(2|D|_{m}\right)^{2 / m}\right\},
$$

and for $d \in(m-1, m)$ and $\lambda>0$,

$$
N_{D}(\lambda) \leq W_{D}(\lambda)+10^{m} C \lambda^{d / 2}\{(m-d)(d-m+1)\}^{-1} .
$$


Remark 2.3.

(i) Note that hypothesis (2.21) implies that $D$ is bounded.

(ii) It is possible to prove upper bounds of the form (2.23), (2.24) assuming (2.2) instead of (2.21), and using [12, Lemma 3.3]. Alternatively, it is possible to obtain (2.23), (2.24) assuming (2.2) and a strong Hardy inequality. See [2] for details and [5, Theorem 5] for a related application.

Proof of Theorem 2.2. Let $a>0$ be such that (2.6) holds, and form the Whitney partition of $D$ into hypercubes of size $\left\{a 2^{-p}: p=0,1,2, \ldots\right\}$. Let $p(\lambda) \in \mathbb{N}$ be the unique integer such that

$$
2^{p(\lambda)} \leq a \lambda^{1 / 2}<2^{p(\lambda)+1} .
$$

This is possible by (2.22) and (2.6). The cubes of size $\left\{a 2^{-p}: p=0,1,2, \ldots\right.$, $p(\lambda)-1\}$ in the Whitney partition cover the set $\{x \in D: \operatorname{dist}(x, \partial D)>$ $\left.a 2^{1-p(\lambda)} m^{1 / 2}\right\}$. We consider the set $\left\{x \in D: \operatorname{dist}(x, \partial D) \leq a 2^{1-p(\lambda)} m^{1 / 2}\right\}$, and we denote by $n_{p(\lambda)}^{\prime}$ the number of hypercubes of size $a 2^{-p(\lambda)}$ that are needed to cover this set. As in the proof of Theorem 2.1 we have $n_{0}=0$,

$$
n_{p} \leq a^{-m} 2^{m p} \mu\left(a 2^{1-p} m^{1 / 2}\right), \quad p=1,2, \ldots, p(\lambda)-1,
$$

and

$$
n_{p(\lambda)}^{\prime} \leq a^{-m} 2^{m p(\lambda)} \omega\left(a 2^{1-p(\lambda)} m^{1 / 2}\right) .
$$

Since $\mu(\varepsilon) \leq \omega(\varepsilon)$ we have

$$
n_{p} \leq a^{-m} 2^{m p} \omega\left(a 2^{1-p} m^{1 / 2}\right), \quad p=1,2, \ldots, p(\lambda) .
$$

Let $\tilde{N}_{a}(\lambda)$ denote the spectral counting function for the Neumann laplacian on a hypercube of size $a$ in $\mathbb{R}^{m}$. Then

$$
\begin{aligned}
& \tilde{N}_{a}(\lambda)=\#\left\{\left(k_{1}, k_{2}, \ldots, k_{m}\right)\right. \in(\mathbb{N} \cup\{0\})^{m}: \\
&\left.\pi^{2}\left\{k_{1}^{2}+\cdots+k_{m}^{2}\right\}<\lambda a^{2}\right\} .
\end{aligned}
$$

By (2.22) and (2.6),

$$
\lambda^{1 / 2} a \geq 2^{1+1 / m} .
$$


Hence by (2.29) and (2.30),

$$
\begin{aligned}
\tilde{N}_{a}(\lambda) & \leq 2^{-m}\left|\left\{x \in \mathbb{R}^{m}:|x|<\frac{\lambda^{1 / 2} a}{\pi}+m^{1 / 2}\right\}\right|_{m} \\
& =(4 \pi)^{-m / 2}\left(\Gamma\left(1+\frac{m}{2}\right)\right)^{-1} a^{m} \lambda^{m / 2}\left\{1+\frac{\pi m^{1 / 2}}{\lambda^{1 / 2} a}\right\}^{m} \\
& \leq(4 \pi)^{-m / 2}\left(\Gamma\left(1+\frac{m}{2}\right)\right)^{-1} a^{m} \lambda^{m / 2}\left\{1+\frac{\pi m^{3 / 2}}{\lambda^{1 / 2} a}\left(1+\frac{\pi m^{1 / 2}}{2^{1+1 / m}}\right)^{m}\right\} .
\end{aligned}
$$

By Neumann bracketing (see e.g. [15, XIII.15]) and by (2.31),

$$
\begin{aligned}
N_{D}(\lambda) \leq & \sum_{p=1}^{p(\lambda)-1} n_{p} \tilde{N}_{a 2^{-p}}(\lambda)+n_{p(\lambda)}^{\prime} \tilde{N}_{a 2^{-p}(\lambda)}(\lambda) \\
\leq & \sum_{p=1}^{p(\lambda)-1} n_{p}(4 \pi)^{-m / 2}\left(\Gamma\left(1+\frac{m}{2}\right)\right)^{-1} a^{m} 2^{-m p} \lambda^{m / 2} \\
& \cdot\left\{1+\frac{\pi m^{3 / 2} 2^{p}}{\lambda^{1 / 2} a}\left(1+\frac{\pi m^{1 / 2}}{2^{1+1 / m}}\right)^{m}\right\}+n_{p(\lambda)}^{\prime} \tilde{N}_{a 2^{-p(\lambda)}}(\lambda) .
\end{aligned}
$$

Note that

$$
\sum_{p=1}^{p(\lambda)-1} n_{p} a^{m} 2^{-m p} \leq|D|_{m} .
$$

Hence by (2.32) and (2.33),

$$
N_{D}(\lambda) \leq W_{D}(\lambda)+B_{1}+B_{2},
$$

where

$$
\begin{aligned}
B_{1}=\sum_{p=1}^{p(\lambda)-1} n_{p} \pi m^{3 / 2} & \left\{\frac{1}{2 \pi^{1 / 2}}+\frac{(\pi m)^{1 / 2}}{2^{2+1 / m}}\right\}^{m} \\
\cdot & \left(\Gamma\left(1+\frac{m}{2}\right)\right)^{-1} a^{m-1} 2^{-(m-1) p} \lambda^{(m-1) / 2},
\end{aligned}
$$

and

$$
B_{2}=n_{p(\lambda)}^{\prime} \tilde{N}_{a 2^{-p(\lambda)}}(\lambda)
$$


By (2.29), and by the definition of $p(\lambda)$,

(2.37) $\tilde{N}_{a 2^{-p(\lambda)}}(\lambda)$

$$
\begin{aligned}
& =\#\left\{\left(k_{1}, \ldots, k_{m}\right) \in(\mathbb{N} \cup\{0\})^{m}: \pi^{2}\left(k_{1}^{2}+\cdots+k_{m}^{2}\right)<\lambda a^{2} 2^{-2 p(\lambda)}\right\} \\
& \leq \#\left\{\left(k_{1}, \ldots, k_{m}\right) \in(\mathbb{N} \cup\{0\})^{m}: \pi^{2}\left(k_{1}^{2}+\cdots+k_{m}^{2}\right)<4\right\}=1 .
\end{aligned}
$$

Hence for $B_{2}$ we obtain, by (2.36), (2.37), (2.28), and (2.21) and by the definition of $p(\lambda)$,

$$
\begin{aligned}
B_{2}=n_{p(\lambda)}^{\prime} & \leq a^{-m} 2^{m p(\lambda)} C\left(a 2^{1-p(\lambda)} \sqrt{m}\right)^{m-d} \\
& =(4 m)^{(m-d) / 2} C\left(a^{-1} 2^{p(\lambda)}\right)^{d} \leq C(4 m)^{(m-d) / 2} \lambda^{d / 2} .
\end{aligned}
$$

To estimate $B_{1}$ we first suppose $d=m-1$. By (2.28), (2.21), and (2.35),

$$
\begin{aligned}
B_{1}= & \sum_{p=1}^{p(\lambda)-1} C 2 \pi m^{2}\left\{\frac{1}{2 \pi^{1 / 2}}+\frac{(\pi m)^{1 / 2}}{2^{2+1 / m}}\right\}^{m}\left(\Gamma\left(1+\frac{m}{2}\right)\right)^{-1} \lambda^{(m-1) / 2} \\
\leq C 2 \pi m^{2} & \left\{\frac{1}{2 \pi^{1 / 2}}+\frac{(\pi m)^{1 / 2}}{2^{2+1 / m}}\right\}^{m} \\
& \cdot(\log 4)^{-1}\left(\Gamma\left(1+\frac{m}{2}\right)\right)^{-1} \lambda^{(m-1) / 2} \log \left\{\left(2|D|_{m}\right)^{2 / m} \lambda\right\}
\end{aligned}
$$

By (2.38) and (2.39) we have, for $d=m-1$ and for $\lambda \geq 4|D|_{m}^{-2 / m}$,

$$
B_{1}+B_{2} \leq C 10^{m} \lambda^{(m-1) / 2} \log \left\{\left(2|D|_{m}\right)^{2 / m} \lambda\right\} .
$$

Suppose $m-1<d<m$. By (2.28), (2.21), and (2.35),

$$
\begin{aligned}
B_{1}=C a^{m-d-1} \lambda^{(m-1) / 2} 2^{m-d} \pi m^{3 / 2}\left\{\frac{1}{2 \pi^{1 / 2}}+\frac{(\pi m)^{1 / 2}}{2^{2+1 / m}}\right\}^{m} \\
\cdot\left(\Gamma\left(1+\frac{m}{2}\right)\right)^{-1} m^{(m-d) / 2} \sum_{p=1}^{p(\lambda)-1} 2^{p(d+1-m)} .
\end{aligned}
$$

By the definition of $p(\lambda)$,

$$
\sum_{p=1}^{p(\lambda)-1} 2^{p(d+1-m)} \leq \frac{2^{(p(\lambda)-1)(d+1-m)}}{1-2^{m-d-1}} \leq \frac{\left(a \lambda^{1 / 2}\right)^{d+1-m}}{2^{d+1-m}-1} .
$$


Combining (2.38), (2.41), and (2.42), we obtain

$$
\begin{aligned}
& B_{1}+B_{2} \leq C \lambda^{d / 2}(4 m)^{1 / 2}\left\{1+\pi m\left(\frac{1}{2 \pi^{1 / 2}}+\frac{(\pi m)^{1 / 2}}{2^{2+1 / m}}\right)^{m}\right. \\
&\left.\cdot\left(\Gamma\left(1+\frac{m}{2}\right)\right)^{-1} \frac{1}{(1+d-m) \log 2}\right\} \\
& \leq 10^{m} C \lambda^{d / 2}(1+d-m)^{-1} .
\end{aligned}
$$

The result now follows by combining (2.40), (2.43), and (2.34).

\section{Proof of TheOREM 1.1 FOR $\alpha<m-1$}

Since $\Omega$ is bounded in $\mathbb{R}^{m-1}(m=3,4, \ldots)$ and $\partial \Omega$ is piecewise smooth, or $\Omega$ is an open, bounded interval in $\mathbb{R}^{1}$, there exist $\Lambda<\infty, v<\infty$, and $\kappa<\infty$ such that

$$
\begin{aligned}
& \Lambda=\max \left\{\left|y-y^{\prime}\right|: y \in \partial \Omega, y^{\prime} \in \partial \Omega\right\}, \\
& \left|N_{\Omega}(\mu)-W_{\Omega}(\mu)\right| \leq v \mu^{(m-2) / 2}, \quad \mu>0, \\
& \mu_{\Omega}(\varepsilon)=|\{y \in \Omega: \operatorname{dist}(y, \partial \Omega)<\varepsilon\}|_{m-1} \leq \kappa \varepsilon, \quad \varepsilon>0 .
\end{aligned}
$$

Assertion (3.2) (or (1.4)) follows from the results in [14], [19].

Theorem 3.1. Suppose $f:[0, \infty) \rightarrow(0,1]$ is monotonically decreasing to zero, right continuous, and $f(0)=1$. Suppose that $\Omega$ is open, bounded and star-shaped with respect to $(0, \ldots, 0)$, and suppose that (3.1)-(3.3) hold with $\Lambda<\infty, v<\infty$, and $\kappa<\infty$. Then for all $L>\Lambda$ and all $\lambda$ sufficiently large (but independent of $L$ ),

$$
\begin{gathered}
\left|N_{D(\Omega, f)}(\lambda)-\pi^{-1} \int_{[0, \infty)} d x \int_{[0, \infty)}\left\{\left(\lambda-\mu(f(x))^{-2}\right)_{+}\right\}^{1 / 2} d N_{\Omega}(\mu)\right| \\
\leq\left(10 \Lambda^{m-2}+6 \kappa+2 v\right) L \lambda^{(m-1) / 2} \log \left(2 \lambda L^{2}\right) \\
+\Lambda^{m-1}(f(L))^{(m-1) / 2} \lambda^{(2 m-1) / 4} \\
\cdot\left\{\int_{\left\{x \geq L: \Lambda f(x) \geq \pi \lambda^{-1 / 2}\right\}} d x(f(x))^{m-1}\right\}^{1 / 2} .
\end{gathered}
$$

Proof. Since $\Omega$ is star-shaped with respect to $(0, \ldots, 0), D(\Omega, f)$ is hornshaped in $\mathbb{R}^{m}$. By [4, Estimate (29)],

$$
\begin{aligned}
\left|N_{D(\Omega, f)}(\lambda)-\pi^{-1} \int_{[0, \infty)} d x \sum_{k=1}^{\infty}\left\{\left(\lambda-\mu_{k}(x)\right)_{+}\right\}^{1 / 2}\right| \\
\quad \leq\left(1+\delta \lambda^{1 / 2} \pi^{-1}\right) N_{\Omega}(\lambda)+\sum_{i=1}^{\infty} \sharp\left\{k \in \mathbb{Z}^{+}: \mu_{k}(i \delta)<\lambda\right\},
\end{aligned}
$$


where $\mu_{k}(x)$ is the $k^{\text {th }}$ eigenvalue of the $(m-1)$-dimensional Dirichlet laplacian on $D(\Omega, f) \cap\left\{(\xi, \eta) \in \mathbb{R}^{m}: \xi=x\right\}$. By scaling we have

$$
\mu_{k}(x)=(f(x))^{-2} \mu_{k} .
$$

By (3.6), and by monotonicity of $f$ and $N_{\Omega}$, we obtain

$$
\begin{aligned}
\sum_{i=1}^{\infty} \sharp\left\{k \in \mathbb{Z}^{+}: \mu_{k}(i \delta)<\lambda\right\} & =\sum_{i=1}^{\infty} N_{\Omega}\left(\lambda(f(i \delta))^{2}\right) \\
& \leq \delta^{-1} \int_{[0, \infty)} N_{\Omega}\left(\lambda(f(x))^{2}\right) d x .
\end{aligned}
$$

The choice

$$
\delta=\pi^{1 / 2} \lambda^{-1 / 4}\left\{N_{\Omega}(\lambda)\right\}^{-1 / 2}\left\{\int_{[0, \infty)} N_{\Omega}\left(\lambda(f(x))^{2}\right) d x\right\}^{1 / 2}
$$

yields, by (3.5), (3.7), and (3.8),

$$
\begin{aligned}
& \left|N_{D(\Omega, f)}(\lambda)-\pi^{-1} \int_{[0, \infty)} d x \int_{[0, \infty)}\left\{\left(\lambda-\mu(f(x))^{-2}\right)_{+}\right\}^{1 / 2} d N_{\Omega}(\mu)\right| \\
& \quad \leq N_{\Omega}(\lambda)+2 \pi^{-1 / 2} \lambda^{1 / 4}\left\{N_{\Omega}(\lambda)\right\}^{1 / 2}\left\{\int_{[0, \infty)} N_{\Omega}\left(\lambda(f(x))^{2}\right) d x\right\}^{1 / 2} .
\end{aligned}
$$

For $L>0$ we define

$$
\begin{aligned}
& D_{L}^{1}=D(\Omega, f) \cap\left\{(x, y) \in \mathbb{R}^{m}: 0<x<L\right\}, \\
& D_{L}^{2}=D(\Omega, f) \cap\left\{(x, y) \in \mathbb{R}^{m}: x>L\right\} .
\end{aligned}
$$

By putting Dirichlet boundary conditions on the set $D(\Omega, f) \cap\{x=L\}$, we obtain

$$
N_{D(\Omega, f)}(\lambda) \geq N_{D_{L}^{1}}(\lambda)+N_{D_{L}^{2}}(\lambda) .
$$

We use Theorem 2.1 to obtain a lower bound for $N_{D_{L}^{1}}$ : define

$$
\mu_{D_{L}^{1}}(\varepsilon)=\left|\left\{z \in D_{L}^{1}: \operatorname{dist}\left(z, \partial D_{L}^{1}\right)<\varepsilon\right\}\right|_{m} .
$$

Lemma 3.2. Suppose $\Omega$ and $f$ satisfy the conditions of Theorem 3.1, (3.1), and (3.3). Then for all $\varepsilon>0$

$$
\mu_{D_{L}^{1}}(\varepsilon) \leq\left(3 \Lambda^{m-1}+L \kappa\right) \varepsilon .
$$


Proof. Note that

(3.14) $\mid\left.\left\{z \in D_{L}^{1}: z=(x, y), x<\varepsilon\right.$ or $\left.x>L-\varepsilon\right\}\right|_{m} \leq 2 \varepsilon|\Omega|_{m-1} \leq 2 \varepsilon \Lambda^{m-1}$.

Let

(3.15) $A=\left\{(x, y) \in D_{L}^{1}\right.$ :

$$
\varepsilon<x<L-\varepsilon, y \in f(x) \Omega, \operatorname{dist}((x, y), \partial D(\Omega, f))<\varepsilon\} .
$$

Suppose $z=(x, y) \in A$. Then there exists $\left(x^{\prime}, y^{\prime}\right) \in \partial D(\Omega, f)$ such that $\left|x-x^{\prime}\right|<\varepsilon$. In fact, since $f$ is monotonically decreasing, $x \leq x^{\prime} \leq x+\varepsilon$. Since $y^{\prime} \in f\left(x^{\prime}\right) \partial \Omega$, it follows that $y^{\prime} \notin f\left(x^{\prime}\right) \Omega$. Hence $y^{\prime} \notin f(x+\varepsilon) \Omega$. Then $y^{\prime} \in f(x) \bar{\Omega} \backslash f(x+\varepsilon) \Omega$, so that $y \in f(x) \bar{\Omega} \backslash f(x+\varepsilon) \Omega$ or $y \in f(x+\varepsilon) \Omega$ and $\operatorname{dist}(y, f(x+\varepsilon) \partial \Omega)<\varepsilon$. Hence

$$
\begin{aligned}
& \int_{A} d z \leq \\
& \quad \leq \int_{[\varepsilon, L-\varepsilon)} d x\left\{|f(x) \bar{\Omega} \backslash f(x+\varepsilon) \Omega|_{m-1}\right. \\
& \left.\quad+|\{y \in f(x+\varepsilon) \Omega: \operatorname{dist}(y, f(x+\varepsilon) \partial \Omega)<\varepsilon\}|_{m-1}\right\} \\
& \leq \int_{[\varepsilon, L-\varepsilon)} d x\left\{\left\{(f(x))^{m-1}-(f(x+\varepsilon))^{m-1}\right\}|\Omega|_{m-1}+\mu_{\Omega}(\varepsilon)\right\} \\
& \leq|\Omega|_{m-1}\left\{\int_{[\varepsilon, 2 \varepsilon)}(f(x))^{m-1} d x-\int_{[L-\varepsilon, L)}(f(x))^{m-1} d x\right\}+\int_{[\varepsilon, L-\varepsilon)} \kappa \varepsilon d x \\
& \leq\left(\Lambda^{m-1}+L \kappa\right) \varepsilon,
\end{aligned}
$$

where we have used various scaling properties and (3.3). The lemma follows by (3.14) and (3.16).

Lemma 3.3. For $L>\Lambda$ and

$$
\begin{gathered}
\lambda \geq 4\left(\Lambda(f(\Lambda))^{m-1}|\Omega|_{m-1}\right)^{-2 / m}, \\
N_{D_{L}^{1}}(\lambda) \geq \pi^{-1} \int_{[0, L)} d x \int_{[0, \infty)}\left\{\left\{\lambda-\mu(f(x))^{-2}\right\}_{+}\right\}^{1 / 2} d N_{\Omega}(\mu) \\
-\left(9 \Lambda^{m-2}+3 \kappa+\nu\right) L \lambda^{(m-1) / 2} \log \left(2 \lambda L^{2}\right) .
\end{gathered}
$$

Proof. For $L>\Lambda$ we have

$$
\left|D_{L}^{1}\right|_{m} \geq \Lambda(f(\Lambda))^{m-1}|\Omega|_{m-1} .
$$


Lemma (3.2) combined with (3.17) and (3.19) guarantee that Theorem 2.1 holds with constants $d=m-1$ and $C=3 \Lambda^{m-1}+L \kappa$. By Theorem 2.1, we thus have that for $L>\Lambda$ and $\lambda$ satisfying (3.17),

$$
N_{D_{L}^{1}}(\lambda) \geq W_{D_{L}^{1}}(\lambda)-\left(9 \Lambda^{m-1}+3 L \kappa\right) \lambda^{(m-1) / 2} \log \left(2 \lambda L^{2}\right) .
$$

Moreover, using (3.2) we obtain the following:

$$
\begin{aligned}
& \frac{1}{\pi} \int_{[0, L)} d x \int_{[0, \infty)}\left\{\left(\lambda-(f(x))^{-2} \mu\right)_{+}\right\}^{1 / 2} d N_{\Omega}(\mu) \\
& \quad=\frac{1}{2 \pi} \int_{[0, L)} d x \int_{\left[0, \lambda(f(x))^{2}\right)}\left(\lambda-(f(x))^{-2} \mu\right)^{-1 / 2}(f(x))^{-2} N_{\Omega}(\mu) d \mu \\
& \quad \leq \frac{1}{2 \pi} \int_{[0, L)} d x \int_{\left[0, \lambda(f(x))^{2}\right)}\left(\lambda-(f(x))^{-2} \mu\right)^{-1 / 2}(f(x))^{-2} \\
& \quad \leq W_{D_{L}^{1}}(\lambda)+v L \lambda^{(m-2) / 2} .
\end{aligned}
$$

By (3.17) and (3.1)

$$
\lambda \geq 4\left(\Lambda|\Omega|_{m-1}\right)^{-2 / m} \geq 4 \Lambda^{-2} \geq 4 L^{-2} .
$$

Hence

$$
v L \lambda^{(m-2) / 2} \leq 2^{-1} v L \Lambda^{(m-1) / 2} \leq v L \lambda^{(m-1) / 2} \log \left(2 \lambda L^{2}\right),
$$

and the Lemma follows by (3.20), (3.21), and (3.23).

In order to obtain a lower bound for $N_{D_{L}^{2}}$ we note that $D_{L}^{2}$ is a region of the form (1.6), with $\Omega$ and $f$ being replaced by $f(L) \Omega$ and $f(x+L) / f(L)$ respectively. Before we use (3.9) we prove the following lemma.

Lemma 3.4. Suppose $\Omega$ satisfies (3.1). Then for all $\lambda>0$,

$$
\begin{gathered}
N_{\Omega}(\lambda) \leq\left[\pi^{-1} \Lambda^{m-1} \lambda^{(m-1) / 2}\right] \leq \pi^{-1} \Lambda^{m-1} \lambda^{(m-1) / 2}, \\
\mu_{1} \geq\left(\frac{\pi}{\Lambda}\right)^{2} .
\end{gathered}
$$


Proof. There exists an $(m-1)$-dimensional hypercube $H$ with volume $\Lambda^{m-1}$ such that $\Omega \subset H$. By monotonicity of the Dirichlet eigenvalues,

$$
\begin{aligned}
N_{\Omega}(\lambda) & \leq N_{H}(\lambda) \\
& =\#\left\{\left(k_{1}, \ldots, k_{m-1}\right) \in \mathbb{Z}_{+}^{m-1}: k_{1}^{2}+\cdots+k_{m-1}^{2}<\frac{\lambda \Lambda^{2}}{\pi^{2}}\right\} \\
& \leq 2^{1-m} \frac{\pi^{(m-1) / 2}}{\Gamma((m+1) / 2)}\left(\frac{\lambda \Lambda^{2}}{\pi^{2}}\right)^{(m-1) / 2} \\
& \leq \pi^{-1} \Lambda^{m-1} \lambda^{(m-1) / 2} .
\end{aligned}
$$

Since $N_{\Omega}(\lambda)$ is an integer, we also have the first inequality in (3.25). Finally

$$
\mu_{1} \geq(m-1)\left(\frac{\pi}{\Lambda}\right)^{2} \geq\left(\frac{\pi}{\Lambda}\right)^{2} .
$$

By (3.9),

$$
\begin{aligned}
& N_{D_{L}^{2}}(\lambda) \\
& \geq \pi^{-1} \int_{[L, \infty)} d x \int_{[0, \infty)}\left\{\left\{\lambda-\mu(f(x))^{-2}\right\}_{+}\right\}^{1 / 2} d N_{\Omega}(\mu)-N_{f(L) \Omega}(\lambda) \\
& \quad-2 \pi^{-1 / 2} \lambda^{1 / 4}\left\{N_{f(L) \Omega}(\lambda) \int_{[0, \infty)} N_{f(L) \Omega}\left(\lambda\left(\frac{f(x+L)}{f(L)}\right)^{2}\right) d x\right\}^{1 / 2} .
\end{aligned}
$$

By Lemma 3.4,

$$
N_{f(L) \Omega}(\lambda)=N_{\Omega}\left(\lambda(f(L))^{2}\right) \leq \pi^{-1} \Lambda^{m-1}(f(L))^{m-1} \lambda^{(m-1) / 2},
$$

and

$$
\begin{aligned}
& \left\{N_{f(L) \Omega}(\lambda) \int_{[0, \infty)} N_{f(L) \Omega}\left(\lambda\left(\frac{f(x+L)}{f(L)}\right)^{2}\right) d x\right\}^{1 / 2} \\
& \quad=\left\{N_{\Omega}\left(\lambda(f(L))^{2}\right) \int_{[L, \infty)} N_{\Omega}\left(\lambda(f(x))^{2}\right) d x\right\}^{1 / 2} \\
& \quad \leq\left\{N_{\Omega}\left(\lambda(f(L))^{2}\right) \int_{\left\{x \geq L, \Lambda f(x) \geq \pi \lambda^{-1 / 2}\right\}} N_{\Omega}\left(\lambda(f(x))^{2}\right) d x\right\}^{1 / 2} \\
& \quad \leq(f(L))^{(m-1) / 2} \lambda^{(m-1) / 2}\left\{\int_{\left\{x \geq L, \Lambda f(x) \geq \pi \lambda^{-1 / 2}\right\}}(f(x))^{m-1} d x\right\}^{1 / 2} .
\end{aligned}
$$


Combining (3.11), Lemma (3.3), and (3.28)-(3.30), we obtain that for $L>\Lambda$ and $\lambda$ large,

$$
\begin{aligned}
N_{D(\Omega, f)}(\lambda) \geq & \pi^{-1} \int_{[0, \infty)} d x \int_{[0, \infty)}\left\{\left\{\lambda-\mu(f(x))^{-2}\right\}_{+}\right\}^{1 / 2} d N_{\Omega}(\mu) \\
& -\left(10 \Lambda^{m-2}+3 \kappa+\nu\right) L \lambda^{(m-1) / 2} \log \left(2 \lambda L^{2}\right) \\
- & \Lambda^{m-1}(f(L))^{(m-1) / 2} \lambda^{(2 m-1) / 4} \\
& \cdot\left\{\int_{\left\{x \geq L, \Lambda f(x) \geq \pi \lambda^{-1 / 2}\right\}}(f(x))^{m-1} d x\right\}^{1 / 2} .
\end{aligned}
$$

To prove the upper bound in Theorem 3.1, we put Neumann boundary conditions on $D(\Omega, f) \cap\{(x, y): x=L\}$. Then

$$
N_{D(\Omega, f)}(\lambda) \leq \tilde{N}_{D_{L}^{1}}(\lambda)+\tilde{N}_{D_{L}^{2}}(\lambda),
$$

where $\tilde{N}_{D_{L}^{1}}, \tilde{N}_{D_{L}^{2}}$ are the counting functions for $D_{L}^{1}, D_{L}^{2}$ with Neumann boundary conditions at $x=L$ and Dirichlet boundary conditions elsewhere. Following [4, Theorem 8 and its proof], (3.5)-(3.9), and (3.28)-(3.30),

$$
\begin{gathered}
\tilde{N}_{D_{L}^{2}}(\lambda) \leq \pi^{-1} \int_{[L, \infty)} d x \int_{[0, \infty)}\left\{\left\{\lambda-\mu(f(x))^{-2}\right\}_{+}\right\}^{1 / 2} d N_{\Omega}(\mu) \\
+\pi^{-1} \Lambda^{m-1}(f(L))^{m-1} \lambda^{(m-1) / 2} \\
+\Lambda^{m-1}(f(L))^{(m-1) / 2} \lambda^{(2 m-1) / 4} \\
\cdot\left\{\int_{\left\{x \geq L, \Lambda f(x) \geq \pi \lambda^{-1 / 2}\right\}}(f(x))^{m-1} d x\right\}^{1 / 2} \cdot \square
\end{gathered}
$$

Lemma 3.5. For $L>\Lambda$ and $\lambda$ sufficiently large (independently of $L$ ),

$$
\begin{array}{r}
\tilde{N}_{D_{L}^{1}}(\lambda) \leq \pi^{-1} \int_{[0, L)} d x \int_{[0, \infty)}\left\{\left\{\lambda-\mu(f(x))^{-2}\right\}_{+}\right\}^{1 / 2} d N_{\Omega}(\mu) \\
+\left(\left(9+\pi^{-1}\right) \Lambda^{m-1}+6 L \kappa+2 \nu L\right) \lambda^{(m-1) / 2} \log \left(2 \lambda L^{2}\right)
\end{array}
$$

Proof. Let $R=\Omega \times(0, L), H_{C}=R \backslash \overline{D_{L}^{1}}$, and denote by $\tilde{N}_{H_{c}}$ the counting function for the Laplace operator for $H_{C}$ with Neumann boundary conditions at $x=L$ and Dirichlet conditions elsewhere. Then

$$
\tilde{N}_{D_{L}^{1}}(\lambda) \leq \tilde{N}_{D_{L}^{1}}(\lambda)+\tilde{N}_{H_{c}}(\lambda)-N_{H_{c}}(\lambda) \leq \tilde{N}_{R}(\lambda)-N_{H_{c}}(\lambda),
$$


where $\tilde{N}_{R}$ is the counting function for the Laplace operator for $R$ with Neumann boundary conditions at $x=L$ and Dirichlet conditions elsewhere. The second inequality in (3.35) follows from the fact that removing the Dirichlet boundary conditions on $\partial D_{L}^{1} \cap \partial H_{C}$ decreases the spectrum, and hence increases the counting function to $\tilde{N}_{R}$. By separation of variables,

$$
\begin{aligned}
\tilde{N}_{R}(\lambda) & =\#\left\{\left(k_{1}, k_{2}\right) \in \mathbb{Z}_{+}^{2}: \mu_{k_{1}}+\frac{\pi^{2}\left(k_{2}-\frac{1}{2}\right)^{2}}{L^{2}}<\lambda\right\} \\
& \leq N_{\Omega}(\lambda)+\sum_{k_{2} \geq 2} N_{\Omega}\left(\lambda-\frac{\pi^{2}\left(k_{2}-\frac{1}{2}\right)^{2}}{L^{2}}\right) .
\end{aligned}
$$

Since $N_{\Omega}$ is monotone,

$$
\begin{aligned}
\tilde{N}_{R}(\lambda) & \leq N_{\Omega}(\lambda)+\int_{[0, \infty)} d x N_{\Omega}\left(\lambda-\frac{\pi^{2} x^{2}}{L^{2}}\right) \\
& =N_{\Omega}(\lambda)+\frac{L}{2 \pi} \int_{[0, \lambda)} d \xi N_{\Omega}(\xi)(\lambda-\xi)^{-1 / 2} .
\end{aligned}
$$

By Lemma (3.4) and (3.2),

(3.38) $\tilde{N}_{R}(\lambda) \leq$

$$
\begin{aligned}
& \leq \pi^{-1} \Lambda^{m-1} \lambda^{(m-1) / 2}+\frac{L}{2 \pi} \int_{[0, \lambda)} d \xi\left\{W_{\Omega}(\xi)+\nu \xi^{(m-2) / 2}\right\}(\lambda-\xi)^{-1 / 2} \\
& =\pi^{-1} \Lambda^{m-1} \lambda^{(m-1) / 2}+W_{R}(\lambda)+\frac{\nu L}{(4 \pi)^{1 / 2}} \cdot \frac{\Gamma(m / 2)}{\Gamma((m+1) / 2)} \lambda^{(m-1) / 2} \\
& \leq W_{R}(\lambda)+\left(\pi^{-1} \Lambda^{m-1}+\nu L\right) \lambda^{(m-1) / 2} .
\end{aligned}
$$

It remains to find a lower bound for $N_{H_{c}}$. In order to apply Theorem 2.1 we need a bound on $\mu_{H_{c}}(\varepsilon)$.

Lemma 3.6. Suppose $\Omega$ and $f$ satisfy the conditions of Theorem 3.1, (3.1), and (3.3). Then

$$
\mu_{H_{c}}(\varepsilon) \leq\left(3 \Lambda^{m-1}+2 L \kappa\right) \varepsilon .
$$

Proof. We note the following:

$$
\begin{aligned}
& \left|\left\{(x, y) \in H_{c}: x \geq L-\varepsilon\right\}\right|_{m} \leq|\Omega|_{m-1} \varepsilon \leq \Lambda^{m-1} \varepsilon, \\
& \left|\left\{(x, y) \in H_{c}: 0<x \leq \varepsilon\right\}\right|_{m} \leq|\Omega|_{m-1} \varepsilon \leq \Lambda^{m-1} \varepsilon, \\
& \left|\left\{(x, y) \in H_{c}: \operatorname{dist}(y, \partial \Omega)<\varepsilon\right\}\right|_{m} \leq L \kappa \varepsilon .
\end{aligned}
$$


Let

$$
\begin{aligned}
B=\left\{(x, y) \in H_{c}: \varepsilon<x<L-\varepsilon, y \in \Omega \backslash\right. & f(x) \Omega, \\
& \operatorname{dist}((x, y), \partial D(\Omega, f)<\varepsilon\} .
\end{aligned}
$$

Suppose $(x, y) \in B$. Then there exists $\left(x^{\prime}, y^{\prime}\right) \in \partial D(\Omega, f)$ such that $\left|x-x^{\prime}\right|<$ $\varepsilon$. Since $f$ is monotonically decreasing, $x-\varepsilon \leq x^{\prime} \leq x$. Since $y^{\prime} \in f\left(x^{\prime}\right) \partial \Omega$, it follows that $y^{\prime} \notin \Omega \backslash f(x-\varepsilon) \bar{\Omega}$. Then $y^{\prime} \in(\bar{\Omega} \backslash f(x) \Omega) \backslash(\Omega \backslash f(x-\varepsilon) \bar{\Omega})$, so that $y \in(\bar{\Omega} \backslash f(x) \Omega) \backslash(\Omega \backslash f(x-\varepsilon) \bar{\Omega})$, or $y \in \Omega \backslash f(x-\varepsilon) \bar{\Omega}$ and dist $(y, f(x-$ $\varepsilon) \partial \Omega)<\varepsilon$. Hence

$$
\begin{aligned}
\int_{B} d z & \leq \int_{[\varepsilon, L-\varepsilon)} d x\left\{|(\bar{\Omega} \backslash f(x) \Omega) \backslash(\Omega \backslash f(x-\varepsilon) \Omega)|_{m-1}\right. \\
& \left.+|\{y \in f(x-\varepsilon) \Omega: \operatorname{dist}(y, f(x-\varepsilon) \partial \Omega)<\varepsilon\}|_{m-1}\right\} \\
& \leq \int_{[\varepsilon, L-\varepsilon)} d x\left\{\left\{(f(x-\varepsilon))^{m-1}-(f(x))^{m}\right\}|\Omega|_{m-1}+\mu_{\Omega}(\varepsilon)\right\} \\
& \leq \varepsilon|\Omega|_{m-1}+(L-2 \varepsilon) \kappa \varepsilon \\
& \leq\left(|\Lambda|^{m-1}+L \kappa\right) \varepsilon .
\end{aligned}
$$

We obtain (3.39) by adding up the contributions from (3.40), (3.41), (3.42), and (3.44).

Since $\left|H_{c}\right|_{m} \rightarrow \infty$ as $L \rightarrow \infty$, we have that for $\lambda$ sufficiently large, $\lambda \geq$ $4\left(\left|H_{c}\right|_{m}\right)^{-2 / m}$. Therefore by Theorem 2.1 , for all $\lambda$ sufficiently large and $L>\Lambda$,

$$
\begin{aligned}
N_{H_{c}}(\lambda) & \geq W_{H_{c}}(\lambda)-3\left(3 \Lambda^{m-1}+2 L \kappa\right) \lambda^{(m-1) / 2} \log \left\{\lambda\left(2\left|H_{c}\right|_{m}\right)^{2 / m}\right\} \\
& \geq W_{H_{c}}(\lambda)-3\left(3 \Lambda^{m-1}+2 L \kappa\right) \lambda^{(m-1) / 2} \log \left(2 \lambda L^{2}\right) .
\end{aligned}
$$

For all $\lambda$ sufficiently large, we have by (3.35), (3.38), and (3.45)

$$
\begin{aligned}
\tilde{N}_{D_{L}^{1}}(\lambda) & \leq W_{R}(\lambda)-W_{H_{c}}(\lambda)+\left(9 \Lambda^{m-1}+6 L \kappa+\nu L\right) \lambda^{(m-1) / 2} \log \left(2 \lambda L^{2}\right) \\
& =W_{D_{L}^{1}}(\lambda)+\left(\left(9+\pi^{-1}\right) \Lambda^{m-1}+6 L \kappa+\nu L\right) \lambda^{(m-1) / 2} \log \left(2 \lambda L^{2}\right) .
\end{aligned}
$$

To complete the proof of Lemma 3.5 we note that by (3.2),

$$
\begin{aligned}
\pi^{-1} \int_{[0, L)} d x \int_{[0, \infty)}\left\{\left(\lambda-\mu(f(x))^{-2}\right)_{+}\right\}^{1 / 2} d N_{\Omega}(\mu) \\
\geq \frac{1}{2 \pi} \int_{[0, L)} d x \int_{\left[0, \lambda(f(x))^{2}\right)}\left(\lambda-(f(x))^{-2} \mu\right)^{-1 / 2}(f(x))^{-2} \\
\cdot\left\{W_{\Omega}(\mu)-v \mu^{(m-2) / 2}\right\} d \mu \quad \geq
\end{aligned}
$$




$$
\geq W_{D_{L}^{1}}(\lambda)-v L \lambda^{(m-1) / 2} .
$$

Lemma 3.5 follows by (3.46) and (3.47).

From (3.33) and (3.34), we obtain that for $L>\Lambda$ and $\lambda$ sufficiently large,

$$
\begin{aligned}
N_{D(\Omega, f)}(\lambda) \leq & \pi^{-1} \int_{[0, \infty)} d x \int_{[0, \infty)}\left\{\left(\lambda-\mu(f(x))^{-2}\right)_{+}\right\}^{1 / 2} d N_{\Omega}(\mu) \\
& +\left(10 \Lambda^{m-2}+6 \kappa+2 v\right) L \lambda^{(m-1) / 2} \log \left(2 \lambda L^{2}\right) \\
& \left.+\Lambda^{m-1}(f(L))^{(m-1) / 2} \lambda^{(2 m-1) / 4}\right) \\
& \cdot\left\{\int_{\left\{x \geq L: \Lambda f(x) \geq \pi \lambda^{-1 / 2}\right\}} d x(f(x))^{m-1}\right\}^{1 / 2} .
\end{aligned}
$$

Theorem 3.1 follows from (3.31) and (3.48).

Remark 3.7. By using (3.2) it is easily seen that, if $D(\Omega, f)$ has finite volume, then the double integral in the left hand side of (3.4) contains the leading (Weyl) term, whereas the right hand side of (3.4) is $O\left(\lambda^{(m-1) / 2} \log \lambda\right)$ with the choice $L=2 \Lambda$. However, in order to extract two-term asymptotics from (3.4) for finite volume horns, we require that $f$ decays in a regular fashion, and not faster than the critical power for $\alpha$ in Theorem 1.1 (v).

Proof of Theorem 1.1 (v) and (vi). Since

$$
f_{\alpha}(L)=(1+L)^{-1 / \alpha},
$$

we obtain that the right hand side of (3.4) is bounded by

$$
c\left\{L \lambda^{(m-1) / 2}+L^{(\alpha+2-2 m) /(2 \alpha)} \lambda^{(2 m-1) / 4}\right\} \log \left(2 \lambda L^{2}\right),
$$

for some constant $c<\infty$. Choose $\lambda$ sufficiently large, and

$$
L=\lambda^{\alpha /(2 \alpha+4 m-4)}
$$

then (3.50) and (3.51) show that the right hand side of (3.4) is

$$
O\left(\lambda^{\left(m \alpha+2 m^{2}-4 m+2\right) /(2 \alpha+4 m-4)}\right) .
$$


Finally, we compute the integral in the left hand side of (3.4):

$$
\begin{aligned}
& \pi^{-1} \int_{[0, \infty)} d x \int_{[0, \infty)}\left\{\left(\lambda-\mu(f(x))^{-2}\right)_{+}\right\}^{1 / 2} d N_{\Omega}(\mu) \\
& =(2 \pi)^{-1} \int_{[0, \infty)} d x(f(x))^{-2} \int_{\left[0, \lambda(f(x))^{2}\right)} d \mu\left(\lambda-\mu(f(x))^{-2}\right)^{-1 / 2} W_{\Omega}(\mu) \\
& +(2 \pi)^{-1} \int_{[1, \infty)} d x x^{2 / \alpha} \int_{\left[0, \lambda x^{-2 / \alpha}\right)} d \mu\left(\lambda-\mu x^{2 / \alpha}\right)^{-1 / 2}\left(N_{\Omega}(\mu)-W_{\Omega}(\mu)\right) .
\end{aligned}
$$

The first term in the right hand side of (3.52) can be computed explicitly, by using (1.3) for the open bounded set $\Omega$ in $\mathbb{R}^{m-1}$, if we make the change of variables $\mu=\xi \lambda(f(x))^{2}$. This yields

$$
(4 \pi)^{-m / 2}\left(\Gamma\left(1+\frac{m}{2}\right)\right)^{-1}|\Omega|_{m-1} \int_{[0, \infty)}(f(x))^{m-1} d x=W_{D(\Omega, f)}(\lambda) .
$$

Suppose $m-1>\alpha>m-2$. We write the second term in the right hand side of (3.52) as

$$
\begin{gathered}
(2 \pi)^{-1} \int_{[0, \infty)} d x x^{2 / \alpha} \int_{\left[0, \lambda x^{-2 / \alpha}\right)} d \mu\left(\lambda-\mu x^{2 / \alpha}\right)^{-1 / 2}\left(N_{\Omega}(\mu)-W_{\Omega}(\mu)\right) \\
-(2 \pi)^{-1} \int_{[0,1)} d x x^{2 / \alpha} \int_{\left[0, \lambda x^{-2 / \alpha}\right)} d \mu\left(\lambda-\mu x^{2 / \alpha}\right)^{-1 / 2}\left(N_{\Omega}(\mu)-W_{\Omega}(\mu)\right) .
\end{gathered}
$$

It is straightforward to check that the first double integral in (3.54) converges absolutely, since $m-2<\alpha<m-1$. By Fubini's theorem this term is equal to $\eta(\alpha, \Omega) \lambda^{(1+\alpha) / 2}$. The second term is bounded in absolute value by

$$
\begin{aligned}
(2 \pi)^{-1} \int_{[0, \lambda)} d \mu & (\lambda-\mu)^{-1 / 2}\left|N_{\Omega}(\mu)-W_{\Omega}(\mu)\right| \\
& \leq(2 \pi)^{-1} \nu \int_{[0, \lambda)} d \mu(\lambda-\mu)^{-1 / 2} \mu^{(m-2) / 2} \leq \nu \lambda^{(m-1) / 2} .
\end{aligned}
$$

Suppose $m>2$ and $\alpha \leq m-2$. The absolute value of the second term in the right-hand side of (3.52) is bounded from above by

$$
\begin{aligned}
(2 \pi)^{-1} \int_{[0, \lambda)} & d \mu \int_{\left[1,(\lambda / \mu)^{\alpha / 2)}\right.} d x x^{2 / \alpha}\left(\lambda-\mu x^{2 / \alpha}\right)^{-1 / 2}\left|N_{\Omega}(\mu)-W_{\Omega}(\mu)\right| \\
& \leq \frac{\alpha}{2 \pi} \lambda^{\alpha / 2} \int_{[0, \lambda)} d \mu \mu^{-(\alpha+2) / 2}(\lambda-\mu)^{1 / 2}\left|N_{\Omega}(\mu)-W_{\Omega}(\mu)\right|
\end{aligned}
$$

The contribution from the interval $\left[0, \mu_{1}\right)$ in $(3.56)$ is $O\left(\lambda^{(1+\alpha) / 2}\right)$, since $N_{\Omega}(\mu)=$ 0 for $0 \leq \mu \leq \mu_{1}$, and $W_{\Omega}$ is given by (1.3). The contribution from the interval 
$\left[\mu_{1}, \lambda\right)$ in (3.56) is, by (3.2), bounded from above by

$$
\frac{\alpha \nu}{2 \pi} \lambda^{(1+\alpha) / 2} \int_{\left[\mu_{1}, \lambda\right)} d \mu \mu^{(m-\alpha-4) / 2}=O\left(\lambda^{(m-1) / 2}\right) .
$$

To complete the proof, we note that $m-1>\left(1-m+[(m-1)(9 m-17)]^{1 / 2}\right) / 2 \geq$ $m-2$, and so we separately consider two cases:

Case I: $m-1>\alpha>\left(1-m+[(m-1)(9 m-17)]^{1 / 2}\right) / 2$,

Case II: $\left(1-m+[(m-1)(9 m-17)]^{1 / 2}\right) / 2>\alpha \geq m-2$.

In Case I, we have that

$$
\frac{1+\alpha}{2}
$$

is strictly greater than both $\left(m \alpha+2 m^{2}-4 m+2\right) /(2 \alpha+4 m-4)$ and $(m-1) / 2$; and so $\eta(\alpha, \Omega) \lambda^{(1+\alpha) / 2}$ is the second term in the asymptotic expansion. In Case II (which can only occur for $m>2$ ), we have that

$$
\frac{m \alpha+2 m^{2}-4 m+2}{2 \alpha+4 m-4}
$$

is greater or equal to both $(1+\alpha) / 2$ and $(m-1) / 2$. This completes the proof of (v) and (vi).

4. PRoOf OF TheOREM 1.1 FOR $\alpha=m-1$

In this section we use Theorem 3.1 to prove Theorem 1.1 for $\alpha=m-1$. Substitution of

$$
f(x)=(1+x)^{-1 /(m-1)}
$$

into the right hand side of (3.4) yields a bound

$$
c\left\{L \lambda^{(m-1) / 2} \log \left(2 \lambda L^{2}\right)+L^{-1 / 2} \lambda^{(2 m-1) / 4)} \log \lambda\right\}
$$

for some $c<\infty$. Choose $\lambda$ sufficiently large and $L=\lambda^{1 / 6}$. Then (4.2) gives the remainder estimate in Theorem 1.1 (iv). To compute the double integral in the left hand side of (3.4), we write

$$
\begin{aligned}
& \pi^{-1} \int_{[0, \infty)} d x \int_{[0, \infty)} d N_{\Omega}(\mu)\left\{\left(\lambda-\mu(1+x)^{2 /(m-1)}\right)_{+}\right\}^{1 / 2} \\
& =\pi^{-1} \int_{[0, \infty)} d x \int_{[0, \lambda)} d N_{\Omega}(\mu)\left\{\left(\lambda-\mu x^{2 /(m-1)}\right)_{+}\right\}^{1 / 2} \\
& \quad-\pi^{-1} \int_{[0, \lambda)} d\left(N_{\Omega}(\mu)-W_{\Omega}(\mu)\right) \int_{[0,1)} d x\left\{\left(\lambda-\mu x^{2 /(m-1)}\right)_{+}\right\}^{1 / 2} \\
& \quad-\pi^{-1} \int_{[0, \lambda)} d W_{\Omega}(\mu) \int_{[0,1)} d x\left\{\left(\lambda-\mu x^{2 /(m-1)}\right)_{+}\right\}^{1 / 2}
\end{aligned}
$$


To compute the third term in the right hand side of (4.3), we make the substitution $x=y^{(m-1) / 2}$, and subsequently

$$
\mu=(\lambda / y) \xi
$$

This gives

$$
\begin{aligned}
(4.4)-\frac{m-1}{2 \pi} \int_{[0, \lambda)} d W_{\Omega}(\mu) \int_{[0,1)} d y y^{(m-3) / 2}(\lambda-\mu y)^{1 / 2} \\
=-\frac{m-1}{2}(4 \pi)^{-m / 2}\left(\Gamma\left(1+\frac{m}{2}\right)\right)^{-1}|\Omega|_{m-1} \\
\cdot\left[\psi\left(\frac{m+2}{2}\right)-\psi\left(\frac{m-1}{2}\right)\right] \lambda^{m / 2},
\end{aligned}
$$

where we have used $[7,4.253 .1]$, and where $\psi$ is defined in $[7,8.360]$. By [7, 8.365.1, 8.365.2, and 8.375.2], we obtain

$$
\begin{aligned}
\psi\left(\frac{m+2}{2}\right)-\psi\left(\frac{m-1}{2}\right) & =\frac{2}{m}+\psi\left(\frac{m}{2}\right)-\psi\left(\frac{m-1}{2}\right) \\
& =2\left[(-1)^{m} \log 2+\sum_{k=1}^{m} \frac{(-1)^{k+m}}{k}+\frac{1}{m-1}\right]
\end{aligned}
$$

To estimate the second term in the right hand side of (4.3) we integrate by parts to obtain

$$
\begin{aligned}
& -(2 \pi)^{-1} \int_{[0, \lambda)} d \mu\left(N_{\Omega}(\mu)-W_{\Omega}(\mu)\right) \\
& \cdot \int_{[0,1)} d x x^{2 /(m-1)}\left(\lambda-\mu x^{2 /(m-1)}\right)^{-1 / 2} \\
& -\pi^{-1}\left(N_{\Omega}(\lambda)-W_{\Omega}(\lambda)\right) \int_{[0,1)} d x\left(\lambda-\lambda x^{2 /(m-1)}\right)^{1 / 2} .
\end{aligned}
$$

The second term in (4.6) is estimated by (3.2) and is $O\left(\lambda^{(m-1) / 2}\right)$. The first term in (4.6) is bounded using (3.2) by

$$
\begin{aligned}
(2 \pi)^{-1} \int_{[0, \lambda)} d \mu\left|N_{\Omega}(\mu)-W_{\Omega}(\mu)\right| \int_{[0,1)} d x x^{2 /(m-1)}\left(\lambda-\mu x^{2 /(m-1)}\right)^{-1 / 2} \\
\quad \leq(2 \pi)^{-1} \int_{[0, \lambda)} d \mu\left|N_{\Omega}(\mu)-W_{\Omega}(\mu)\right|(\lambda-\mu)^{-1 / 2} \\
\quad=O\left(\lambda^{(m-1) / 2}\right) .
\end{aligned}
$$


Applying Fubini's Theorem to the first term in the right hand side of (4.3) gives

$$
\begin{aligned}
\pi^{-1} \int_{[0, \lambda)} d N_{\Omega}(\mu) \int_{\left[0,(\lambda / \mu)^{(m-1) / 2}\right)} d x\left(\lambda-\mu x^{2 /(m-1)}\right)^{1 / 2} \\
=(4 \pi)^{-m / 2}\left(\Gamma\left(\frac{m+2}{2}\right)\right)^{-1}|\Omega|_{m-1} \lambda^{m / 2} \\
+(4 \pi)^{-1 / 2} \Gamma\left(\frac{m+1}{2}\right)\left(\Gamma\left(\frac{m+2}{2}\right)\right)^{-1} \frac{m-1}{2} \lambda^{m / 2} \\
\cdot \int_{\left[\mu_{1}, \lambda\right)} \mu^{-(m+1) / 2} W_{\Omega}(\mu) d \mu \\
+(4 \pi)^{-1 / 2} \Gamma\left(\frac{m+1}{2}\right)\left(\Gamma\left(\frac{m+2}{2}\right)\right)^{-1}\left(N_{\Omega}(\lambda)-W_{\Omega}(\lambda)\right) \lambda^{1 / 2} \\
+(4 \pi)^{-1 / 2} \Gamma\left(\frac{m+1}{2}\right)\left(\Gamma\left(\frac{m+2}{2}\right)\right)^{-1} \frac{m-1}{2} \lambda^{m / 2} \\
\cdot \int_{\left[\mu_{1}, \infty\right)} \mu^{-(m+1) / 2}\left(N_{\Omega}(\mu)-W_{\Omega}(\mu)\right) d \mu \\
-(4 \pi)^{-1 / 2} \Gamma\left(\frac{m+1}{2}\right)\left(\Gamma\left(\frac{m+2}{2}\right)\right)^{-1} \frac{m-1}{2} \lambda^{m / 2} \\
\quad \int_{[\lambda, \infty)} \mu^{-(m+1) / 2}\left(N_{\Omega}(\mu)-W_{\Omega}(\mu)\right) d \mu .
\end{aligned}
$$

The second term in the right hand side of (4.8) is equal to

$$
(4 \pi)^{-m / 2}\left(\Gamma\left(\frac{m+2}{2}\right)\right)^{-1} \frac{m-1}{2}|\Omega|_{m-1} \lambda^{m / 2} \log \frac{\lambda}{\mu_{1}} .
$$

The third and fifth terms in the right hand side of (4.8) can be estimated by (3.2) and are both $O\left(\lambda^{(m-1) / 2}\right)$. To complete the proof of (1.14) we combine (4.4), (4.5), (4.8), and (4.9).

\section{Proof of Theorem 1.1 For $\alpha>m-1$}

Theorem 5.1. Suppose $f:[0, \infty) \rightarrow(0,1]$ is monotonically decreasing to 0 , right continuous, and $f(0)=1$. Suppose that $\Omega$ is an open, bounded set in $\mathbb{R}^{m-1}$, and star-shaped with respect to $(0, \ldots, 0)$. Suppose that (3.1)-(3.3) hold for $\Lambda<\infty$, $v<\infty$, and $\kappa<\infty$. Let $0=L_{0}<L_{1}<\cdots<L_{K+1}=\infty$ for some non-negative integer $K$. Then 


$$
\begin{gathered}
\left|N_{D(\Omega, f)}(\lambda)-\pi^{-1} \int_{[0, \infty)} d x \int_{[0, \infty)}\left\{\left(\lambda-\mu(f(x))^{-2}\right)_{+}\right\}^{1 / 2} d N_{\Omega}(\mu)\right| \\
\leq \sum_{i=1}^{K+1} \Lambda^{m-1}\left\{\lambda^{(m-1) / 2}\left(f\left(L_{i-1}\right)\right)^{m-1}+3 \lambda^{(2 m-1) / 4}\left(f\left(L_{i-1}\right)\right)^{(m-1) / 2}\right. \\
\left.\cdot\left\{\int_{\left\{L_{i-1} \leq x<L_{i}, f(x) \geq \Lambda^{-1} \lambda^{-1 / 2}\right\}} d x(f(x))^{m-1}\right\}^{1 / 2}\right\} .
\end{gathered}
$$

Proof. Define

$$
D_{i}(\Omega, f)=D(\Omega, f) \cap\left\{(x, y) \in \mathbb{R}^{m}: L_{i-1}<x<L_{i}\right\} .
$$

The open set $D_{i}$ is a one-sided horn-shaped open set in $\mathbb{R}^{m}$ with $\mu_{1}(x)=\infty$ for $x>L_{i}$. By [4, Theorem 8], we have for each of the $D_{i}$

$$
\begin{aligned}
\left|N_{D_{i}(\Omega, f)}(\lambda)-\pi^{-1} \int_{\left[L_{i-1}, L_{i}\right)} d x \int_{[0, \infty)}\left\{\left(\lambda-\mu(f(x))^{-2}\right)_{+}\right\}^{1 / 2} d N_{\Omega}(\mu)\right| \\
\leq\left(1+\delta \lambda^{1 / 2} \pi^{-1}\right) \sharp\left\{k \in \mathbb{Z}^{+}: \mu_{k}\left(L_{i-1}\right)<\lambda\right\} \\
+\sum_{\left\{j \in \mathbb{Z}^{+}: L_{i-1}+j \delta<L_{i}\right\}} \sharp\left\{k \in \mathbb{Z}^{+}: \mu_{k}\left(L_{i-1}+j \delta\right)<\lambda\right\} .
\end{aligned}
$$

By Lemma 3.4,

$$
\delta \lambda^{1 / 2} \pi^{-1} \sharp\left\{k \in \mathbb{Z}^{+}: \mu_{k}\left(L_{i-1}\right)<\lambda\right\} \leq \delta \lambda^{m / 2} \Lambda^{m-1}\left(f\left(L_{i-1}\right)\right)^{m-1},
$$

and

$$
\begin{aligned}
& \quad \sum_{\left\{j \in \mathbb{Z}^{+}: L_{i-1}+j \delta<L_{i}\right\}} \#\left\{k \in \mathbb{Z}^{+}: \mu_{k}\left(L_{i-1}+j \delta\right)<\lambda\right\} \\
& \quad \leq \sum_{\left\{j \in \mathbb{Z}^{+}: L_{i-1}+j \delta<L_{i}, \lambda\left(f\left(L_{i-1}+j \delta\right)\right)^{2}>\mu_{1}\right\}} N_{\Omega}\left(\lambda\left(f\left(L_{i-1}+j \delta\right)\right)^{2}\right) \\
& \leq \Lambda^{m-1} \lambda^{(m-1) / 2} \sum_{\left\{j \in \mathbb{Z}^{+}: L_{i-1}+j \delta<L_{i}, \lambda\left(f\left(L_{i-1}+j \delta\right)\right)^{2}>\mu_{1}\right\}}\left(f\left(L_{i-1}+j \delta\right)\right)^{m-1} \\
& \leq \Lambda^{m-1} \lambda^{(m-1) / 2} \delta^{-1} \int_{\left\{L_{i-1}<x<L_{i}, f(x) \geq \Lambda^{-1} \lambda^{-1 / 2}\right\}}(f(x))^{m-1} d x .
\end{aligned}
$$

The choice

$$
\begin{aligned}
\delta=\left(f\left(L_{i-1}\right)\right)^{-(m-1) / 2} \lambda^{-1 / 4} & \cdot\left\{\int_{\left\{L_{i-1}<x<L_{i}, f(x) \geq \Lambda^{-1} \lambda^{-1 / 2}\right\}}(f(x))^{m-1} d x\right\}^{1 / 2}
\end{aligned}
$$


yields

$$
\begin{gathered}
\left|N_{D_{i}(\Omega, f)}(\lambda)-\pi^{-1} \int_{\left[L_{i-1}, L_{i}\right)} d x \int_{[0, \infty)}\left\{\left(\lambda-\mu(f(x))^{-2}\right)_{+}\right\}^{1 / 2} d N_{\Omega}(\mu)\right| \\
\leq \lambda^{(m-1) / 2} \Lambda^{m-1}\left(f\left(L_{i-1}\right)\right)^{m-1}+2 \lambda^{(2 m-1) / 4} \Lambda^{m-1}\left(f\left(L_{i-1}\right)\right)^{(m-1) / 2} \\
\cdot\left\{\int_{\left\{L_{i-1}<x<L_{i}, f(x) \geq \Lambda^{-1} \lambda^{-1 / 2}\right\}}(f(x))^{m-1} d x\right\}^{1 / 2} .
\end{gathered}
$$

By Dirichlet bracketing,

$$
N_{D(\Omega, f)}(\lambda) \geq \sum_{i=1}^{K+1} N_{D_{i}(\Omega, f)}(\lambda),
$$

which yields the lower bound in (5.1) after summing (5.7) over $i=1, \ldots, K+1$.

To prove the upper bound in Theorem 5.1 we denote by $\tilde{N}_{D_{i}(\Omega, f)}$ the counting function for the Laplace operator on $D_{i}(\Omega, f)$ with Neumann boundary conditions at $x=L_{i-1}$ and $x=L_{i}$, and Dirichlet boundary conditions elsewhere. First suppose that $\lambda\left(L_{i}-L_{i-1}\right)^{2}<\pi^{2}$. Since $D_{i}(\Omega, f) \subset f\left(L_{i-1}\right) \Omega \times\left(L_{i-1}, L_{i}\right)$, we have by Lemma 3.4,

$$
\begin{aligned}
\tilde{N}_{D_{i}(\Omega, f)}(\lambda) & \leq N_{\Omega}\left(\left(f\left(L_{i-1}\right)\right)^{2} \lambda\right) \tilde{N}_{\left(L_{i-1}, L_{i}\right)}(\lambda) \\
& =N_{\Omega}\left(\left(f\left(L_{i-1}\right)\right)^{2} \lambda\right) \\
& \leq \lambda^{(m-1) / 2} \Lambda^{m-1}\left(f\left(L_{i-1}\right)\right)^{m-1} \\
& \leq \pi^{-1} \int_{\left[L_{i-1}, L_{i}\right)} d x \int_{[0, \infty)}\left\{\left(\lambda-\mu(f(x))^{-2}\right)_{+}\right\}^{1 / 2} d N_{\Omega}(\mu) \\
& +\lambda^{(m-1) / 2} \Lambda^{m-1}\left(f\left(L_{i-1}\right)\right)^{m-1},
\end{aligned}
$$

where $\tilde{N}_{\left(L_{i-1}, L_{i}\right)}$ is the counting function for an interval $\left(L_{i-1}, L_{i}\right)$ in $\mathbb{R}$ with Neumann boundary conditions at the endpoints. Next suppose that $\lambda\left(L_{i}-L_{i-1}\right)^{2} \geq$ $\pi^{2}$. Fix a positive integer $M$ and let

$$
\begin{aligned}
\delta & =\frac{L_{i}-L_{i-1}}{M}, \\
D_{i}^{+}(\Omega, f)=\{(x, y) & \left.\in \mathbb{R}^{m}: L_{i-1}<x<L_{i}, y \in f^{+}(x) \Omega\right\},
\end{aligned}
$$

where $f^{+}$is the piecewise constant approximation of $f$ defined by

$$
f^{+}(x)=f\left(L_{i-1}+\left[\frac{x-L_{i-1}}{\delta}\right] \delta\right) .
$$


By putting additional Neumann boundary conditions at

$$
x=L_{i-1}+j \delta, \quad j=1, \ldots, M-1
$$

(if $M \geq 2$ ) and following [4, Estimate (30) in proof of Theorem 8], we obtain

$$
\begin{aligned}
& \bar{N}_{D_{i}^{+}(\Omega, f)}(\lambda) \\
\leq & \sum_{j=0}^{M-1} \sharp\left\{(k, \ell) \in\left(\mathbb{Z}^{+} \cup\{0\}\right) \times \mathbb{Z}^{+}: \frac{\pi^{2} k^{2}}{\delta^{2}}+\left(f^{+}\left(L_{i-1}+j \delta\right)\right)^{-2} \mu_{\ell}<\lambda\right\} \\
\leq & \#\left\{k \in \mathbb{Z}^{+} \cup\{0\}: \frac{\pi^{2} k^{2}}{\delta^{2}}<\lambda\right\} \cdot \#\left\{\ell \in \mathbb{Z}^{+}: \mu_{\ell}<\lambda\left(f^{+}\left(L_{i-1}\right)\right)^{2}\right\} \\
& +\sum_{j=1}^{M-1} \#\left\{\ell \in \mathbb{Z}^{+}: \mu_{\ell}<\lambda\left(f^{+}\left(L_{i-1}+j \delta\right)\right)^{2}\right\} \\
& +\sum_{j=1}^{M-1} \#\left\{(k, \ell) \in \mathbb{Z}^{+} \times \mathbb{Z}^{+}: \frac{\pi^{2} k^{2}}{\delta^{2}}+\left(f^{+}\left(L_{i-1}+j \delta\right)\right)^{-2} \mu_{\ell}<\lambda\right\} \\
\leq & \left(1+\delta \lambda^{1 / 2} \pi^{-1}\right) \pi^{-1} \Lambda^{m-1}\left(f\left(L_{i-1}\right)\right)^{m-1} \lambda^{(m-1) / 2} \\
& +\sum_{j=1}^{M-1}\left[\pi^{-1}\left(f\left(L_{i-1}+j \delta\right)\right)^{m-1} \Lambda^{m-1} \lambda^{(m-1) / 2}\right] \\
& +\int_{\left[L_{i-1}, L_{i}\right)} d x \int_{[0, \infty)}\left\{\left(\lambda-\mu(f(x))^{-2}\right)_{+}\right\}^{1 / 2} d N_{\Omega}(\mu) \\
\leq & \left(1+\delta \lambda^{1 / 2}\right) \Lambda^{m-1}\left(f\left(L_{i-1}\right)\right)^{m-1} \lambda^{(m-1) / 2} \\
& +\delta^{-1} \int_{\left[L_{i-1}, L_{i}\right)}\left[\pi^{-1}(f(x))^{m-1} \Lambda^{m-1} \lambda^{(m-1) / 2}\right] d x \\
& +\int_{\left[L_{i-1}, L_{i}\right)} d x \int_{[0, \infty)}\left\{\left(\lambda-\mu(f(x))^{-2}\right)_{+}\right\}^{1 / 2} d N_{\Omega}(\mu) \\
\leq & \left(1+\delta \lambda^{1 / 2}\right) \Lambda^{m-1}\left(f\left(L_{i-1}\right)\right)^{m-1} \lambda^{(m-1) / 2} \\
& +\delta^{-1} \Lambda^{m-1} \lambda^{(m-1) / 2} \int_{\left\{L_{i-1}<x<L_{i}, f(x) \geq \Lambda^{-1} \lambda^{-1 / 2}\right\}}(f(x))^{m-1} d x \\
& +\int_{\left[L_{i-1}, L_{i}\right)} d x \int_{[0, \infty)}\left\{\left(\lambda-\mu(f(x))^{-2}\right)_{+}\right\}^{1 / 2} d N_{\Omega}(\mu) . \\
& \\
&
\end{aligned}
$$

We wish to minimize the right hand side of (5.13) by choosing $\delta$-or equivalently $M$ - appropriately. Let 
(5.14) $\quad M=\left[\lambda^{1 / 4}\left(L_{i}-L_{i-1}\right)\left(f\left(L_{i-1}\right)\right)^{(m-1) / 2}\right.$

$$
\left.\cdot\left\{\int_{\left\{L_{i-1}<x<L_{i}, f(x) \geq \Lambda^{-1} \lambda^{-1 / 2}\right\}}(f(x))^{m-1} d x\right\}^{-1 / 2}\right] .
$$

Since

$$
\begin{aligned}
\left\{\int_{\left\{L_{i-1}<x<L_{i}, f(x) \geq \Lambda^{-1} \lambda^{1 / 2}\right\}}(f(x))^{m-1} d x\right\}^{1 / 2} & \\
\leq & \left(f\left(L_{i-1}\right)\right)^{(m-1) / 2}\left(L_{i}-L_{i-1}\right)^{1 / 2},
\end{aligned}
$$

we conclude that

$$
M \geq\left[\lambda^{1 / 4}\left(L_{i}-L_{i-1}\right)^{1 / 2}\right] \geq\left[\pi^{1 / 2}\right]=1 .
$$

Since $x \geq[x] \geq x / 2$ for $x \geq 1$,

$$
\begin{aligned}
& \delta \leq 2 \lambda^{-1 / 4}\left(f\left(L_{i-1}\right)\right)^{-(m-1) / 2} \\
& \cdot\left\{\int_{\left\{L_{i-1}<x<L_{i}, f(x) \geq \Lambda^{-1} \lambda^{-1 / 2}\right\}}(f(x))^{m-1} d x\right\}^{1 / 2} \leq 2 \delta .
\end{aligned}
$$

Combining (5.13) and (5.17), we obtain for $\lambda\left(L_{i}-L_{i-1}\right)^{2} \geq \pi^{2}$

$$
\begin{aligned}
\tilde{N}_{D_{i}^{+}(\Omega, f) \leq} & \lambda^{(m-1) / 2} \Lambda^{m-1}\left(f\left(L_{i-1}\right)\right)^{m-1} \\
& +3\left(f\left(L_{i-1}\right)\right)^{(m-1) / 2} \Lambda^{m-1} \lambda^{(2 m-1) / 4} \\
& \cdot\left\{\int_{\left\{L_{i-1}<x<L_{i}, f(x) \geq \Lambda^{-1} \lambda^{-1 / 2}\right\}}(f(x))^{m-1} d x\right\}^{1 / 2} \\
& +\pi^{-1} \int_{\left[L_{i-1}, L_{i}\right)} d x \int_{[0, \infty)}\left\{\left(\lambda-\mu(f(x))^{-2}\right)_{+}\right\}^{1 / 2} d N_{\Omega}(\mu) .
\end{aligned}
$$

By Neumann bracketing,

(5.19) $N_{D(\Omega, f)}(\lambda)$

$$
\leq \sum_{\left\{i: \lambda\left(L_{i}-L_{i-1}\right)^{2}<\pi^{2}\right\}} \tilde{N}_{D_{i}(\Omega, f)}(\lambda)+\sum_{\left\{i: \lambda\left(L_{i}-L_{i-1}\right)^{2} \geq \pi^{2}\right\}} \tilde{N}_{D_{i}^{+}(\Omega, f)}(\lambda),
$$

and the upper bound in Theorem 5.1 follows from (5.9), (5.18), and (5.19). 
We now turn to the proof of (1.11)-(1.13) in Theorem 1.1. We first show how the two leading terms occur in (1.11)-(1.13).

Lemma 5.2. Let $f_{\alpha}$ be given by (1.7), and suppose that $\Omega$ is an open, bounded set in $\mathbb{R}^{m-1}$ which satisfies (3.1) and (3.2) with $\Lambda<\infty$ and $v<\infty$. Then for $\alpha>m-1$,

$$
\begin{aligned}
& \pi^{-1} \int_{[0, \infty)} d x \int_{[0, \infty)}\left\{\left(\lambda-\mu\left(f_{\alpha}(x)\right)^{-2}\right)_{+}\right\}^{1 / 2} d N_{\Omega}(\mu) \\
& =\zeta(\alpha, \Omega) \lambda^{(1+\alpha) / 2}-\frac{\alpha}{\alpha+1-m}(4 \pi)^{-m / 2}\left(\Gamma\left(1+\frac{m}{2}\right)\right)^{-1}|\Omega|_{m-1} \lambda^{m / 2} \\
& +O\left(\lambda^{(m-1) / 2}\right) .
\end{aligned}
$$

Proof.

$$
\begin{aligned}
& \pi^{-1} \int_{[0, \infty)} d x \int_{[0, \infty)}\left\{\left(\lambda-\mu\left(f_{\alpha}(x)\right)^{-2}\right)_{+}\right\}^{1 / 2} d N_{\Omega}(\mu) \\
& =\pi^{-1} \int_{[0, \infty)} d x \int_{[0, \infty)}\left\{\left(\lambda-\mu x^{2 / \alpha}\right)_{+}\right\}^{1 / 2} d N_{\Omega}(\mu) \\
& -\pi^{-1} \int_{[0,1)} d x \int_{[0, \infty)}\left\{\left(\lambda-\mu x^{2 / \alpha}\right)_{+}\right\}^{1 / 2} d W_{\Omega}(\mu) \\
& -(2 \pi)^{-1} \int_{[0,1)} d x x^{2 / \alpha} \int_{[0, \infty)}\left(N_{\Omega}(\mu)-W_{\Omega}(\mu)\right) \\
& \cdot\left\{\left(\lambda-\mu x^{2 / \alpha}\right)_{+}\right\}^{-1 / 2} d \mu .
\end{aligned}
$$

Using (3.2), we can bound the absolute value of the third term in the right hand side of (5.21) by

$$
\begin{aligned}
& \int_{[0,1)} d x x^{2 / \alpha} \int_{[0, \infty)}\left|N_{\Omega}(\mu)-W_{\Omega}(\mu)\right|\left\{\left(\lambda-\mu x^{2 / \alpha}\right)_{+}\right\}^{-1 / 2} d \mu \\
& \quad \leq \int_{[0, \infty)}\left|N_{\Omega}(\mu)-W_{\Omega}(\mu)\right|\left\{(\lambda-\mu)_{+}\right\}^{-1 / 2} d \mu \\
& \quad \leq v \int_{[0, \lambda)} \mu^{(m-2) / 2}(\lambda-\mu)^{-1 / 2} d \mu \\
& \quad=O\left(\lambda^{(m-1) / 2}\right) .
\end{aligned}
$$

By Fubini's theorem, we obtain for the first term in the right hand side of (5.21) 


$$
\begin{aligned}
& \pi^{-1} \int_{[0, \infty)} d N_{\Omega}(\mu) \int_{[0, \infty)}\left\{\left(\lambda-\mu x^{2 / \alpha}\right)_{+}\right\}^{1 / 2} d x \\
& \quad=\pi^{-1} \int_{[0, \infty)} d N_{\Omega}(\mu) \int_{[0,(\lambda / \mu) \alpha / 2)}\left(\lambda-\mu x^{2 / \alpha}\right)^{1 / 2} d x \\
& \quad=(4 \pi)^{-1 / 2} \frac{\Gamma(\alpha / 2+1)}{\Gamma(\alpha / 2+3 / 2)} \int_{[0, \infty)} \mu^{-\alpha / 2} d N_{\Omega}(\mu) \lambda^{(1+\alpha) / 2} \\
& \quad=\zeta(\alpha, \Omega) \lambda^{(1+\alpha) / 2} .
\end{aligned}
$$

Note that by Lemma 3.4,

$$
\int_{[0, \infty)} \mu^{-\alpha / 2} d N_{\Omega}(\mu) \leq \Lambda^{-\alpha} \sum_{j=1}^{\infty} j^{-\alpha /(m-1)}<\infty,
$$

since $\alpha>m-1$. To compute the second term in the right hand side of (5.21) we use (1.3) for the open, bounded set $\Omega$ in $\mathbb{R}^{m-1}$ :

$$
W_{\Omega}(\mu)=(4 \pi)^{-(m-1) / 2}\left(\Gamma\left(\frac{m+1}{2}\right)\right)^{-1}|\Omega|_{m-1} \lambda^{(m-1) / 2} .
$$

The second term now becomes

$$
\begin{aligned}
&-\frac{m-1}{2 \pi}|\Omega|_{m-1}(4 \pi)^{-(m-1) / 2}\left(\Gamma\left(\frac{m+1}{2}\right)\right)^{-1} \\
& \cdot \int_{[0,1)} d x \int_{\left[0, \lambda / x^{2 / \alpha}\right)}\left(\lambda-\mu x^{2 / \alpha}\right)^{1 / 2} \mu^{(m-3) / 2} d \mu,
\end{aligned}
$$

which yields the second term in the right hand side of (5.20) after the change of variables $\mu=\xi \lambda x^{-2 / \alpha}$.

To complete the proof of Theorem 1.1 for $\alpha>m-1$, we let

$$
0<\gamma_{1}<\cdots<\gamma_{K},
$$

and put (if $K \geq 1$ )

$$
L_{i}=\lambda^{\gamma_{i}}, \quad i=1, \ldots, K .
$$

The first term in the right hand side of (5.1) is bounded from above by

$$
\sum_{i=1}^{K+1} \lambda^{(m-1) / 2} \Lambda^{m-1}\left(f\left(L_{i-1}\right)\right)^{(m-1) / 2} \leq(K+1) \Lambda^{m-1} \lambda^{(m-1) / 2} .
$$


The $i^{\text {th }}$ term $(K \geq i \geq 2)$ in the second part of the right hand side of (5.1) is bounded from above by

$$
\begin{aligned}
& 3 \lambda^{(2 m-1) / 4} \Lambda^{m-1}\left(1+\lambda^{\gamma_{i-1}}\right)^{-(m-1) /(2 \alpha)} \\
& \cdot\left\{\int_{\left[\lambda^{\left.\gamma_{i-1}, \lambda^{\gamma_{i}}\right)}\right.}(1+x)^{-(m-1) / \alpha} d x\right\}^{1 / 2} \\
& \leq c_{1}(\alpha, m) \Lambda^{m-1} \lambda^{(2 m-1) / 4-\gamma_{i-1}(m-1) /(2 \alpha)+\gamma_{i}(\alpha+1-m) /(2 \alpha),},
\end{aligned}
$$

for all $\lambda \geq 1$ and some constant $c_{1}(\alpha, m)$ depending upon $\alpha$ and $m$ only. Since $L_{K+1}=\infty$, the $(K+1)^{\prime}$ th term in the second part of the right hand side of $(5.1)$ is bounded from above by

$$
c_{2}(\alpha, m) \Lambda^{m-1} \lambda^{(\alpha+m) / 4-(m-1) \gamma_{K} /(2 \alpha)},
$$

for some constant $c_{2}(\alpha, m)$. Finally the term with $i=1$ in the second part of the right-hand side of (5.1) is bounded by

$$
\begin{aligned}
& 3 \Lambda^{m-1} \lambda^{(2 m-1) / 4}\left\{\int_{\left[0, L_{1}\right)}(1+x)^{-(m-1) / \alpha}\right\}^{1 / 2} \\
& \quad \leq c_{3}(\alpha, m) \Lambda^{m-1} \lambda^{(2 m-1) / 4-\gamma_{0}(m-1) /(2 \alpha)+\gamma_{1}(\alpha+1-m) /(2 \alpha)},
\end{aligned}
$$

where we have put $\gamma_{0}=0$. Let $\beta>0$ and choose $\gamma_{1}, \ldots, \gamma_{K}$ such that

$$
\frac{2 m-1}{4}-\frac{(m-1) \gamma_{i-1}}{2 \alpha}+\frac{(\alpha+1-m) \gamma_{i}}{2 \alpha}=\beta, \quad i=1, \ldots, K .
$$

Since $\gamma_{0}=0$,

$$
\gamma_{i}=\sum_{\ell=o}^{i-1}\left(\frac{\alpha+1-m}{m-1}\right)^{-\ell} \frac{\alpha\left(2 \beta-m+\frac{1}{2}\right)}{\alpha+1-m}, \quad i=1, \ldots, K .
$$

Hence

$$
\gamma_{K}=\sum_{\ell=0}^{K-1}\left(\frac{\alpha+1-m}{m-1}\right)^{-\ell} \frac{\alpha\left(2 \beta-m+\frac{1}{2}\right)}{\alpha+1-m} .
$$

We choose $\beta$ such that the $(K+1)^{\prime \text { th }}$ term estimated in (5.31) is also $O\left(\lambda^{\beta}\right)$. This gives the following equation for $\beta$ :

$$
\frac{\alpha+m}{4}-\left(\beta-\frac{m}{2}+\frac{1}{4}\right) \sum_{\ell=0}^{K-1}\left(\frac{m-1}{\alpha+1-m}\right)^{\ell+1}=\beta .
$$


Hence

$$
\begin{aligned}
& \beta=\left\{\frac{\alpha+m}{4}+\frac{2 m-1}{4} \sum_{\ell=0}^{K-1}\left(\frac{m-1}{\alpha+1-m}\right)^{\ell+1}\right\} \\
& \cdot\left\{1+\sum_{\ell=0}^{K-1}\left(\frac{m-1}{\alpha+1-m}\right)^{\ell+1}\right\}^{-1}
\end{aligned}
$$

We note that, since $\alpha>m-1, \beta$ is a decreasing function of $K$, and that by (5.1) and (5.29)-(5.32), the right hand side in (5.1) is bounded from above by

$$
\Lambda^{m-1}(K+1)\left\{\lambda^{(m-1) / 2}+\left(c_{1}(\alpha, m)+c_{2}(\alpha, m)+c_{3}(\alpha, m)\right) \lambda^{\beta}\right\} .
$$

Since $\beta>(m-1) / 2$, the problem of minimizing (5.38) is reduced to minimizing $(K+1) \lambda^{\beta}$ as a function of $K$. We consider the various cases for $\alpha$ below:

If $m-1<\alpha \leq 2 m-2$, then

$$
\beta \leq \frac{2 m-1}{4}+4^{-1}(m+\alpha)\left\{\sum_{\ell=0}^{K-1}\left(\frac{m-1}{\alpha+1-m}\right)^{\ell+1}\right\}^{-1} \leq \frac{2 m-1}{4}+\frac{m}{K}
$$

We choose for $\lambda>1$

$$
K=[m \log \lambda]
$$

By (5.38)-(5.40), we obtain the bound $O\left(\lambda^{(2 m-1) / 4} \log \lambda\right)$ as advertised in (1.13). If $\alpha>2 m-2$, then

$$
\beta \leq \frac{\alpha+1}{4}+\frac{m-1}{4}\left(\frac{m-1}{\alpha+1-m}\right)^{K} .
$$

For $\lambda$ sufficiently large, we choose

$$
K=\left[\frac{\log \log \lambda}{\log ((\alpha+1-m) /(m-1))}\right]
$$

By (5.38), (5.41), and (5.42) we obtain the bound $O\left(\lambda^{(\alpha+1) / 4} \log \log \lambda\right)$ as advertised in (1.11) and (1.12). This completes the proof of Theorem 1.1.

\section{REFERENCES}

[1] M. Abramowitz \& I.A. Stegun, Pocketbook of Mathematical Functions, Harri Deutsch Verlag, Thun, 1984.

[2] A. ANCONA, On strong barriers and an inequality of Hardy for domains in $\mathbb{R}^{n}$, J. Lond. Math. Soc. 34 (1986), 274-290. 
[3] M. VAN DEN BERG, On the spectrum of the Dirichlet laplacian for horn-shaped regions in $\mathbb{R}^{n}$ with infinite volume, J. Funct. Anal. 58 (1984), 150-156.

[4] _ Dirichlet-Neumann bracketing for horn-shaped regions, J. Funct. Anal. 104 (1992), 110120.

[5] - On the spectral counting function for the Dirichlet laplacian, J. Funct. Anal. 107 (1992), 352-361.

[6] R. Courant \& D. Hilbert, Methods of Mathematical Physics I, J. Wiley \& Sons, New York, 1989.

[7] I.S. Gradshteyn \& I.M. Ryzhik, Table of Integrals, Series and Products, Academic Press, New York, 1980.

[8] V. IVRII, Microlocal Analysis and Precise Spectral Asymptotics, Springer, Berlin 1998.

[9] V. JAKŠIĆ, S. MOLČANOV \& B. SIMON, Eigenvalue asymptotics of the Neumann Laplacian of regions and manifolds with cusps, J. Funct. Anal. 106 (1992), 59-79.

[10] M. LIANANTONAKIS, On the eigenvalue counting function for weighted Laplace-Beltrami operators, J. of Geometric Analysis 10 (2000), 299-322.

[11] V.G. MAZ'JA, Polyharmonic capacity in the theory of the first boundary-value problem, Sibirsk. Mat. Ż. 6 (1965), 127-148.

[12] G. MÉTIVIER, Valeurs propres de problèmes aux limites elliptiques irréguliers, Bull. Soc. Math. France Mém. 51-52 (1977), 125-219.

[13] L.B. PARNOVSKI, Asymptotics of Dirichlet spectrum on some class of non-compact domains, Math. Nachr. 174 (1995), 253-263.

[14] PHAM THE LAI, Meilleures éstimations asymptotiques des reste de la fonction spectrale et des valeurs propres rélatifs au laplacien, Math. Scand. 48 (1981), 5-38.

[15] M. ReEd \& B. Simon, Methods of Mathematical Physics IV, Analysis of Operators, Academic Press, New York, 1978.

[16] G.V. RozenbluUm, On the distribution of eigenvalues of the first boundary value problem in unbounded regions, Dokl. Akad. Nauk. SSSR. 200 (1971), 1539-1542.

[17] _ On the eigenvalues of the first boundary value problem in unbounded domains, Math. USSR-Sb. 18 (1972), 235-248.

[18] - The computation of the spectral asymptotics for the Laplace operator in domains of infinite measure, J. Sov. Math. 6 (1976), 64-71.

[19] R. SEELEY, A sharp asymptotic remainder estimate for the eigenvalues of the laplacian in a domain of $\mathbb{R}^{3}$, Adv. Math. 29 (1978), 244-269.

[20] B. SimON, Functional Integration and Quantum Physics, Academic Press, New York, 1979.

[21] _ Nonclassical eigenvalue asymptotics, J. Funct. Anal. 53 (1983), 84-98.

[22] H. TAMURA, The asymptotic distribution of eigenvalues of the Laplace operator in an unbounded domain, Nagoya Math. J. 60 (1976), 7-33.

[23] Y.G. SAFAROV \& D.G. VASSILIEV, The Asymptotic Distribution of Eigenvalues of Partial Differential Operators Translation of Mathematical Monographs Volume 155, American Mathematical Society, 1997.

[24] H. WEYL, Das asymptotische Verteilungsgesetz der Eigenwerte linearer partieller Differentialgleichungen, Math. Ann. 71 (1912), 441-479.

M. VAN DEN BERG:

School of Mathematics

University of Bristol

Bristol, BS8 1TW, England, UK
M. LIANANTONAKIS:

3 Cobden Road, Edinburgh, EH9 2BJ

Scotland, UK

ACKNOWLEDGMENT: The research of ML was supported by UK EPSRC Grant J32084.

American Mathematical Society SubjeCt Classification: 35P20.

Received: December 24th, 1999; revised: July 5th, 2000. 